\title{
菏 University
}

Lu, D., Liang, J., Du, X., Ma, C. and Gao, Z. (2019) Fractional elastoplastic constitutive model for soils based on a novel 3D fractional plastic flow rule. Computers and

Geotechnics, 105, pp. 277-290. (doi:10.1016/j.compgeo.2018.10.004)

There may be differences between this version and the published version. You are advised to consult the publisher's version if you wish to cite from it.

http://eprints.gla.ac.uk/171128/

Deposited on: 11 October 2018

Enlighten - Research publications by members of the University of Glasgow http://eprints.gla.ac.uk 


\title{
Fractional elastoplastic constitutive model for soils based on a novel
} 3D fractional plastic flow rule

\author{
De-chun $\mathrm{LU}^{1}$; Jing-yu LIANG ${ }^{2}$, Xiu-li DU*, Chao $\mathrm{MA}^{4}$, Zhi-wei GAO
}

\section{ABSTRACT:}

A novel three-dimensional (3D) fractional plastic flow rule that is not limited by the coordinate basis of the differentiable function is proposed based on the fractional derivative and the coordinate transformation. By introducing the 3D fractional plastic flow rule into the characteristic stress space, a 3D fractional elastoplastic model for soil is established for the first time. Only five material parameters with clear physical significance are required in the proposed model. The capability of the model in capturing the strength and deformation behaviour of soils under true 3D stress conditions is verified by comparing model predictions with test results.

Keywords: Soils, Constitutive model, Fractional plastic flow rule, True 3D stress condition, Critical state, Characteristic stress.

${ }^{1}$ Ph.D., Professor. Key Laboratory of Urban Security and Disaster Engineering of Ministry of Education, Beijing University of Technology, Beijing, 100124, China. E-mail: dechun@bjut.edu.cn.

${ }^{2}$ Ph.D., Candidate. Key Laboratory of Urban Security and Disaster Engineering of Ministry of Education, Beijing University of Technology, Beijing, 100124, China. E-mail: liangjingyuy@163.com.

3* Ph.D., Professor. Key Laboratory of Urban Security and Disaster Engineering of Ministry of Education, Beijing University of Technology, Beijing, 100124, China (Corresponding author). E-mail: duxiuli5@126.com.

${ }^{4}$ Ph.D., Beijing Advanced Innovation Center for Future Urban Design, Beijing University of Civil Engineering and Architecture, Beijing, 102616, China. E-mail: machao@bucea.edu.cn.

5 Ph.D., School of Engineering, University of Glasgow, Glasgow, G12 8LT, UK. E-mail: zhiwei.gao@glasgow.ac.uk. 


\section{Introduction}

Fractional derivative can generalise integer derivatives to all real (and even complex) orders, and thus provides a way to extend the gradient into the fractional gradient. In contrast to the gradient, the fractional gradient is not orthogonal with the surface of the differentiable function. The ability of the fractional derivative to adjust gradient direction was utilized by Sumelka (2014) to describe the direction of the viscoplastic strain increments, and thus the concept of the fractional plastic flow rule was originally proposed (Sumelka, 2014; Sumelka and Nowak, 2016). The plastic strain increment direction can be obtained by the fractional partial derivative of the yield function with respect to the Cauchy's stress. However, in order to obtain the explicit solutions of the fractional derivative of the yield function, the yield function has to be directly expressed in terms of the principal stresses (Sumelka, 2014) or the stress components (Sumelka and Nowak, 2016), because the chain rule with the same fractional order for the fractional partial derivative does not work. The performance of the fractional plastic flow rule in modelling mechanical behaviour of granular soils in triaxial compression condition has been explored by Sun and Xiao (2017). A series of fractional order plastic models have been established (Sun and Xiao, 2017; Sun et al., 2017) by combining the fractional plastic flow rule with plastic modulus in existing models (Li and Dafalias, 2000; Xiao et $a l ., 2004)$ to describe stress-dilatancy and strain hardening/softening of granular soils. Furthermore, Sun et al. $(2018 \mathrm{a} ; 2018 \mathrm{~b})$ originally used the current stress state in the definition of the fractional derivative to model the state-dependent behaviour of granular soils. However, the fractional plastic flow rule adopted in these models were all derived by the fractional derivative of the yield function with respect to two stress invariants, i.e., the mean stress $p$ and the deviatoric stress $q$ under triaxial compression conditions, rather than the general stress tensor. The existing fractional elastoplastic models for soils suits only for triaxial compression conditions, and the strength and deformation behaviour of soils under true 3D stress conditions has not been described. Indeed, the application of the fractional derivative in elastoplastic constitutive modelling for soils is still at the very beginning.

A 3D yield function and the fractional plastic flow rule, which should be matched, are essential for establishing a 3D fractional constitutive model for soils. Up to the present, a yield function in the 3D stress space can be constructed using two methods: $(i)$ Direct method in which the yield function is explicitly expressed in terms of the stress components (Borja et al., 2003; Khalili and Liu, 2008; Duriez and Vincens, 2015; Panteghini and Lagioia, 2018); (ii) Indirect method where the 
yield function is constructed under triaxial compression conditions in a transitional space, which can reflect yield properties of soils under true 3D stress conditions (Chowdhury and Nakai, 1998; Collins, 2003; Yao et al., 2009; Ma et al., 2017). However, by applying the existing fractional flow rule (Sumelka, 2014) to the commonly used 3D yield functions constructed by the above methods, explicit expressions of the fractional derivative with respect to general stress components $\sigma_{i j}$ cannot be obtained. Thus, a 3D fractional plastic flow rule with no limitation of the coordinate basis of the yield function is eagerly needed for establishing 3D fractional plasticity model.

In this paper, a novel 3D fractional plastic flow rule that can well match the classical plasticity theory is presented based on the covariant transformation. Furthermore, the characteristic stress $(\mathrm{Lu}$ et al., 2017; Ma et al., 2017) is introduced to describe the mechanical behaviours of soil under true 3D stress conditions. The combination of the new 3D fractional plastic flow rule and the characteristic stress presents a new approach to establishing a 3D fractional elastoplastic constitutive model. The strength and deformation behaviour of soils under true 3D stress conditions can thus be directly described.

\section{A novel 3D fractional plastic flow rule}

The plastic flow rule in classical plasticity theory is used to determine the direction of the plastic strain increment. The plastic strain increment direction is usually determined by the orthogonality with respect to the plastic potential surface. If the plastic potential function is chosen to be identical to the yield function, the associated flow rule is adopted (Wood, 1990; Sun et al., 2004; Yao et al., 2009; Zhou and Sheng, 2015). However, the associated flow rule does not work for most geomaterials (Lade et al., 1987; Collins and Houlsby, 1997). Therefore, the non-associated flow rule is usually used (Lu et al., 2016; Gao and Zhao, 2017). There are two approaches to obtaining the desired plastic strain increment direction $\mathbf{n}$ : $(i)$ constructing a plastic potential function $g$ to determine $\mathbf{n}$ by orthogonality as shown in Fig. 1(a); (ii) determining $\mathbf{n}$ based on an existing yield function $f$ in a non-orthogonal way as shown in Fig. 1(b).

Fig. 1 Two approaches to determining the plastic strain increment direction:

(a) orthogonal way; (b) non-orthogonal way. 
However, the plastic potential function may be difficult to construct in a simple way and additional parameters could be required. The fractional partial derivative poses the ability to adjust the gradient direction based on an existing function in a non-orthogonal way. Moreover, the yield curve is necessary for the plastic theory. Therefore, fractional partial derivatives of the yield function is a proper option as the non-orthogonal way for determining the plastic strain increment direction (Sumelka and Nowak, 2016; Sun and Xiao, 2017).

\subsection{Fractional derivative}

There are three widely used definitions for fractional derivatives, i.e., Grünwald-Letnikov, Riemann-Liouville, and Caputo definition (Podlubny, 1998; Mashayekhi et al., 2018). Among them, the Caputo derivative has the weak singularity and the Caputo derivative of a constant is 0 , making it the widely used one (Podlubny, 1998; Meng et al., 2016; Colinas-Armijo et al., 2016;). The Caputo derivative used in this paper is expressed as:

$$
{ }_{a}^{C} D_{x}^{\mu} f(x)=\frac{1}{\Gamma(n-\mu)} \int_{a}^{x} \frac{f^{(n)}(\tau)}{(x-\tau)^{\mu+1-n}} \mathrm{~d} \tau, \quad(x>a)
$$

where ${ }^{C} D^{\mu}$ means the Caputo derivative of order $\mu . a$ is the starting point of integral, which is chosen as 0 in this paper. $x$ is the independent variable, which is used to express the current stress state in this paper. $n$ is the smallest integer greater than $\mu, n=[\mu]+1$, where [-] denotes the floor function. $n=1$ when $0<\mu \leq 1 . \Gamma(\cdot)$ is Euler Gamma function, which is expressed as $\Gamma(z)=\int_{0}^{\infty} e^{-\tau} \tau^{z-1} \mathrm{~d} \tau$, the real part of $z$ is larger than 0 , i.e., $\operatorname{Re}(z)>0$. The explicit expression of the Caputo derivative of a power function $f(x)=x^{m}$ can be obtained as follows:

$$
{ }_{0}^{C} D_{x}^{\mu}\left(x^{m}\right)=\frac{\partial^{\mu}\left(x^{m}\right)}{\partial x^{\mu}}=\frac{\Gamma(m+1)}{\Gamma(m+1-\mu)} x^{m-\mu}
$$

where the exponent $m(>-1)$ is restricted by the integrability of $f(x)$.

When the fractional derivative is used to get the partial derivatives of a function, the fractional gradient can be obtained and it is flexible to adjust the flow direction by adjusting the fractional order $\mu$. This is exactly what is desired for the non-orthogonal way mentioned above to determine the plastic strain increment direction.

\subsection{A 3D fractional plastic flow rule}

In the classical plastic theory, the plastic strain increment direction is determined by the 
gradient of the plastic potential function $g$. The plastic strain increment is expressed as follows:

$$
\mathrm{d} \varepsilon_{i j}^{\mathrm{p}}=\mathrm{d} \lambda \frac{\partial g}{\partial \sigma_{i j}}
$$

where $\mathrm{d} \lambda$ is the plastic multiplier for general plastic flow rule, and $\partial g / \partial \sigma_{i j}$ is the gradient of the plastic potential function, respectively. The plastic potential function can be constructed using the principal stresses $\sigma_{i}(i=1,2,3)$, which is expressed as $g\left(\sigma_{i}\right)$. The gradient direction of $g\left(\sigma_{i}\right)$ in the $\sigma_{i}$ coordinate system is thus expressed by $n_{i}^{(\sigma)}=\partial g / \partial \sigma_{i}$. The plastic potential function of soils can also be constructed by any set of stress variables $S_{k}(k=1,2,3)$, where $S_{k}$ is a function of $\sigma_{i}$, i.e., $S_{k}\left(\sigma_{i}\right)$. The plastic potential function can be expressed as $g\left(S_{\mathrm{k}}\right)$ in the $S_{k}$ coordinate system, and its gradient direction is $n_{k}^{(S)}=\partial g / \partial S_{k}$. Here, $n_{k}^{(S)}$ and $n_{i}^{(\sigma)}$ are components of the gradient vector $\mathbf{n}$ in the $S_{k}$ and $\sigma_{i}$ coordinate systems, respectively, as shown in Fig. 2. Therefore, a covariant transformation coefficient $l_{i}^{k}$ is needed to transform the components of the vector $\mathbf{n}$ in the $S_{k}$ coordinate system into the $\sigma_{i}$ coordinate system. $l_{i}^{k}$ is the partial derivative of $S_{k}$ with respect to $\sigma_{i}$, and is expressed as follows:

$$
l_{i}^{k}=\frac{\partial S_{k}}{\partial \sigma_{i}}
$$

Fig. 2 Coordinate transformation of the plastic strain increment direction

Therefore, according to the covariant transformation rule, $n_{i}^{(\sigma)}$ in the $\sigma_{i}$ coordinate system can be expressed in terms of $n_{k}^{(S)}$ in the $S_{k}$ coordinate system.

$$
n_{i}^{(\sigma)}=n_{k}^{(S)} l_{i}^{k}=n_{k}^{(S)} \frac{\partial S_{k}}{\partial \sigma_{i}}
$$

where $S_{k}$ is an interim coordinate system for specific purposes, like the Cartesian rectangular coordinate system, the cylindrical coordinate system or the spherical coordinate system etc. The commonly used stress invariants $p, q$ and $\theta$ or other stress invariants, like $I_{1}, I_{2}$ and $I_{3}$, are specific examples of $S_{k}$. Generally, the components of the gradient vector of $g$ can be expressed as $n_{k l}^{(S)}=\partial g / \partial S_{k l}$ in the $S_{k l}$ space and $n_{i j}^{(\sigma)}=\partial g / \partial \sigma_{i j}$ in the $\sigma_{i j}$ space, respectively. Therefore, the general covariant transformation between $n_{k l}^{(S)}$ and $n_{i j}^{(\sigma)}$ can be accomplished by the covariant transformation coefficient $l_{i}^{k} l_{j}^{l}=\partial S_{k l} / \partial \sigma_{i j}$ as follows: 


$$
n_{i j}^{(\sigma)}=n_{k l}^{(S)} l_{i}^{k} l_{j}^{l}=n_{k l}^{(S)} \frac{\partial S_{k l}}{\partial \sigma_{i j}}
$$

For geomaterials, stress invariants $p, q$ and $\theta$ are usually adopted to establish constitutive models (Mortara, 2015). The corresponding strain increment $\left(\mathrm{d} \varepsilon_{\mathrm{v}}^{\mathrm{p}}, \mathrm{d} \varepsilon_{\mathrm{s}}^{\mathrm{p}}, \mathrm{d} \varepsilon_{\theta}^{\mathrm{p}}\right)$ can be obtained as follows:

$$
\left(\mathrm{d} \varepsilon_{\mathrm{v}}^{\mathrm{p}}, \mathrm{d} \varepsilon_{\mathrm{s}}^{\mathrm{p}}, \mathrm{d} \varepsilon_{\theta}^{\mathrm{p}}\right)=\mathrm{d} \lambda\left(n_{p}, n_{q}, n_{\theta}\right)
$$

where $\left(n_{p}, n_{q}, n_{\theta}\right)=(\partial g / \partial p, \partial g / \partial q, \partial g / \partial \theta)$ represents the plastic strain increment direction. Two orthogonal planes, i.e., the meridian plane and the deviatoric plane, are used to analyse characteristics of models (Khalili and Liu, 2008; Yao et al., 2009; Lu et al., 2018). The current stress state point $A$ can thus be represented in the meridian plane with the stress Lode angle $\theta$ ( $\theta$ is equivalent to the intermediated principal stress coefficient $b$, namely, $b=2 \tan \theta /(\sqrt{3}+\tan \theta))$ and in the deviatoric plane with $p=p_{A}$, as shown in Fig. 3(a) and (b), respectively. The plastic strain increment direction of point $A$ can also be divided into two components: the vector $\mathbf{n}_{\mathbf{m g}}=\left(n_{p}, n_{q}\right)$ in the meridian plane and the vector $\mathbf{n}_{\mathbf{d} g}=\left(n_{q}, n_{\theta}\right)$ in the deviatoric plane, which are orthogonal to the plastic potential curves as shown in Fig. 3 (a) and (b), respectively. Further, the obtained plastic strain increment direction in the $(p, q, \theta)$ coordinate system can be transformed into the $\sigma_{i j}$ space. The transformation is accomplished by $\partial p / \partial \sigma_{i j}, \partial q / \partial \sigma_{i j}$ and $\partial \theta / \partial \sigma_{i j}$, which is a common practice in the classical elastoplastic theory as follows:

$$
\mathrm{d} \varepsilon_{i j}^{\mathrm{p}}=\mathrm{d} \lambda\left(n_{p} \frac{\partial p}{\partial \sigma_{i j}}+n_{q} \frac{\partial q}{\partial \sigma_{i j}}+n_{\theta} \frac{\partial \theta}{\partial \sigma_{i j}}\right)
$$

Fig. 3 Plastic strain increment direction in: (a) the meridian plane with $\theta=\theta_{A}$; (b) the deviatoric plane with $p=p_{A}$.

The fractional derivative can thus be a new tool and a proper approach to extending the classical plastic flow rule. Sumelka (2014) proposed a concept of fractional plastic flow rule and used it as a new tool to introduce material heterogeneity/multi-scale effects into the constitutive model.

$$
\mathrm{d} \varepsilon_{i j}^{\mathrm{p}}=\Lambda \frac{\partial^{\mu} f}{\partial \sigma_{i j}{ }^{\mu}}
$$


where $\Lambda$ is the plastic multiplier for the fractional plastic flow rule. $\partial^{\mu} f / \partial \sigma_{i j}{ }^{\mu}$ is fractional plastic strain increment direction which is represented by the fractional partial derivative of the yield function $f$ with respective to $\sigma_{i j}$. The flow rule becomes associated when $\mu=1$. However, the explicit expression of the flow direction in the 3D stress space determined by Eq. (9) can only be derived by the yield function directly expressed by $\sigma_{i j}$, such as the Huber-Mises-Hencky yield function (Sumelka, 2014; Sumelka and Nowak, 2016). For geomaterials, the yield function $f$ is usually and more conveniently expressed in terms of the stress invariants $p, q$ and $\theta$ or other stress components for true 3D stress conditions. That is, the constraint of Eq. (9) on the coordinate basis of the yield function limits the application of the fractional plastic flow rule in the form of Eq. (9).

A novel fractional plastic flow rule that suitable for the true 3D stress conditions can be established based on the view of the coordinate transformation rather the chain rule with the same fractional order for the fractional derivative. The proposed fractional plastic flow rule is not limited by the coordinate basis of the yield function and can be obtained in two steps:

(i) Determine the plastic strain increment direction $m_{k l}^{(S)}$ in the $S_{k l}$ space by the fractional partial derivative of the yield function with respect to $S_{k l}$. Therefore, the components of the plastic strain increment can be determined in the $S_{k l}$ space as follows:

$$
\mathrm{d} \varepsilon_{k l}^{\mathrm{p}(S)}=\Lambda m_{k l}^{(S)}=\Lambda \frac{\partial^{\mu} f}{\partial S_{k l}{ }^{\mu}}
$$

(ii) Transform the determined plastic strain components in the $S_{k l}$ space into the $\sigma_{i j}$ space by the covariant transformation coefficient $l_{i}^{k} l_{j}^{l}=\partial S_{k l} / \partial \sigma_{i j}$. Eventually, a novel 3D fractional plastic flow rule in the $\sigma_{i j}$ space is obtained as follows:

$$
\mathrm{d} \varepsilon_{i j}^{\mathrm{p}(\sigma)}=l_{i}^{k} l_{j}^{l} \mathrm{~d} \varepsilon_{k l}^{\mathrm{p}(S)}=\Lambda \frac{\partial^{\mu} f}{\partial S_{k l}{ }^{\mu}} \frac{\partial S_{k l}}{\partial \sigma_{i j}}
$$

It is crucial to transform the determined plastic strain increment direction into the $\sigma_{i j}$ space. Based on the proposed fractional plastic flow rule Eq. (11), the general form of the plastic strain increments under the true 3D stress conditions can be expressed by the stress tensor $\sigma_{i j}$, which are actually essential for soil property analysis and model application. That is, the general form of the fractional plastic flow rule is essential and make it possible to establish a 3D fractional constitutive model for geomaterials in the framework of the plastic theory. 

plastic flow rule can be written as:

$$
\mathrm{d} \varepsilon_{i j}^{\mathrm{p}}=\Lambda \frac{\partial^{\mu} f}{\partial S_{k}^{\mu}} \frac{\partial S_{k}}{\partial \sigma_{i j}}
$$

where $\partial^{\mu} f / \partial S_{k}^{\mu}$ is the determined plastic strain increment direction in the $S_{k}$ coordinate system, which can be transformed into the $\sigma_{i j}$ space by $\partial S_{k} / \partial \sigma_{i j}$. When the commonly used stress invariants $p, q$ and $\theta$ are chosen to construct the yield function, the 3D fractional plastic flow rule becomes:

$$
\mathrm{d} \varepsilon_{i j}^{\mathrm{p}}=\Lambda\left(m_{p} \frac{\partial p}{\partial \sigma_{i j}}+m_{q} \frac{\partial q}{\partial \sigma_{i j}}+m_{\theta} \frac{\partial \theta}{\partial \sigma_{i j}}\right)
$$

The plastic strain increment direction is thus first expressed by $\left(m_{p}, m_{q}, m_{\theta}\right)=\left(\partial^{\mu} f / \partial p^{\mu}\right.$, $\left.\partial^{\mu} f / \partial q^{\mu}, \partial^{\mu} f / \partial \theta^{\mu}\right)$ in the $(p, q, \theta)$ coordinate system, and then transformed into the $\sigma_{i j}$ space by $\partial p / \partial \sigma_{i j}, \partial q / \partial \sigma_{i j}$ and $\partial \theta / \partial \sigma_{i j}$. The plastic strain increment direction represented by $\left(m_{p}, m_{q}, m_{\theta}\right)$ can be divided into two components $\mathbf{m}_{\mathbf{m} \mu}=\left(m_{p}, m_{q}\right)$ and $\mathbf{m}_{\mathbf{d} \mu}=\left(m_{q}, m_{\theta}\right)$ in two orthogonal planes, i.e., the meridian plane and the deviatoric plane. $\mathbf{m}_{\mathbf{m} \mu}$ and $\mathbf{m}_{\mathbf{d} \mu}$ are represented by the red solid arrows, as shown in Fig. 4. When $\mu=1$, the fractional gradient directions degenerate to the normal direction of the yield curve, which can be decomposed into $\mathbf{n}_{\mathbf{m} f}$ and $\mathbf{n}_{\mathbf{d} f}$, indicated by the blue solid arrows. The deviation degree of the fractional gradient direction from the normal of the yield curve can be adjusted by $\mu$.

In particular, when the yield function is expressed only by $q$ and $\theta$ and is independent of $p$ for the hydrostatic pressure independent materials, the yield curve in the meridian plane is a straight line that is parallel to the $p$-axis, as shown in Fig. 4(c). Therefore, the fractional derivative of $f$ with respect to $p$ is 0 and is independent of $\mu$. The plastic strain increment direction becomes $\left(0, m_{q}, m_{\theta}\right)$. $\mathbf{m}_{\mathbf{m} \mu}$ is perpendicular to the yield line in the meridian plane. Nonetheless, $\mathbf{m}_{\mathbf{d} \mu}$ still depends on $\mu$, which is similar to the effects of $\mu$ on $\mathbf{m}_{\mathbf{d} \mu}$ in Fig. 4(b). When the yield function is expressed only by $p$ and $q$ and is independent of $\theta$, the yield curve in the deviatoric plane is a circle as shown in Fig. 4(d). Then the fractional derivative of $f$ with respect to $\theta$ is 0 and is independent of $\mu$. The plastic strain increment direction can be represented by $\left(m_{p}, m_{q}, 0\right)$. $\mathbf{m}_{\mathbf{d} \mu}$ is perpendicular to the yield circle in the deviatoric plane. However, $\mathbf{m}_{\mathbf{m} \mu}$ still depends on $\mu$, which is similar to the effects of $\mu$ on $\mathbf{m}_{\mathbf{m} \mu}$ in Fig. 4(a). 
Fig. 4 Fractional plastic strain increment direction with the yield function constructed:

(a) by $p, q$ and $\theta$ in the meridian plane; (b) by $p, q$ and $\theta$ in the deviatoric plane;

(c) only by $q$ and $\theta$ in the meridian plane; (d) only by $p$ and $q$ in the deviatoric plane.

\section{7}

The proposed 3D fractional plastic flow rule offers a new approach to determine the plastic strain increment directions under true 3D stress conditions. Therefore, a new possibility is provided for establishing true 3D fractional elastoplastic constitutive model for soils.

\section{A 3D fractional elastoplastic model in the characteristic stress space}

In order to describe soil behaviour under true $3 \mathrm{D}$ stress conditions in a simple way, some specialized stress concepts were proposed, like the $t_{i j}$ stress (Chowdhury and Nakai, 1998, Nakai et al., 2011), the transformed stress (Yao et al., 2004; Sun et al., 2004; Yao et al., 2009) and the characteristic stress (Lu et al., 2017; Ma et al., 2017) etc. The yield function $\hat{f}$ can be constructed using only two common stress invariants $\hat{p}$ and $\hat{q}$ in a chosen specialized stress space, which is able to directly describe soil behaviour under true 3D stress conditions. That is, the $3 \mathrm{D}$ constitutive model can be directly established under triaxial compression conditions in the specialized stress space. The new proposed fractional plastic flow rule becomes simpler and more powerful by combining with a specialized stress space, and Eq. (13) becomes:

$$
\mathrm{d} \varepsilon_{i j}^{\mathrm{p}}=\Lambda\left(\frac{\partial^{\mu} \hat{f}}{\partial \hat{p}^{\mu}} \frac{\partial \hat{p}}{\partial \hat{\sigma}_{i j}}+\frac{\partial^{\mu} \hat{f}}{\partial \hat{q}^{\mu}} \frac{\partial \hat{q}}{\partial \hat{\sigma}_{i j}}\right)
$$

The characteristic stress space is a proper choice to describe the soil behaviour especially for the true 3D stress conditions (Lu et al., 2017; Ma et al., 2017). The combination of the characteristic stress and the fractional derivative is elaborately attempted and discussed in this paper.

\subsection{Characteristic stress}

The concept of the characteristic stress, which was originally proposed by Lu et al. (2017) presents a new approach to describing the soil behaviour under true 3D stress conditions (Ma et al., 2017). The principal values of $\sigma_{i j}$ that is denoted by $\hat{\sigma}_{m n}(m, n=1,2,3)$ can be obtained by the coordinate transformation (Chowdhury and Nakai, 1998) in the $\sigma_{i j}$ space as follows: 


$$
\hat{\sigma}_{m n}=Q_{m i} Q_{n j} \sigma_{i j}=\left\{\begin{array}{cc}
\sigma_{m} & m=n \\
0 & m \neq n
\end{array}\right.
$$

where $Q_{i j}$ is an orthogonal tensor which transforms the general stress tensor to its principal values. The principal stress tensor of the characteristic stress in this paper can be expressed by the following equation:

$$
\hat{c}_{m n}=\left\{\begin{array}{cc}
p_{r}\left(\frac{\sigma_{m}}{p_{r}}\right)^{\beta} & m=n \\
0 & m \neq n
\end{array}\right.
$$

where $p_{\mathrm{r}}$ is the reference stress for dimensionless transformation, and $p_{\mathrm{r}}=1 \mathrm{kPa}$ is usually used. $\beta$ is a material parameter and varies from 0 to 1 for different geomaterials. The characteristic principal stress is coaxial with the general principal stress. Finally, the characteristic stress tensor can be obtained by the following coordinate transformation in the $c_{i j}$ space:

$$
c_{i j}=Q_{i m} Q_{j n} \hat{c}_{m n}
$$

The comparison of stress variables in the $\sigma_{i j}$ space and the $c_{i j}$ space is listed in Tab. 1, in which $\varphi_{\mathrm{c}}$ is

\begin{tabular}{|c|c|c|}
\hline & General stress space & Characteristic stress space \\
\hline Stress tensor & $\sigma_{i j}$ & $c_{i j}$ \\
\hline Principal stress & $\sigma_{i}, \quad i=1,2,3$ & $c_{i}=p_{r}\left(\frac{\sigma_{i}}{p_{r}}\right)^{\beta}, \quad i=1,2,3$ \\
\hline Mean stress & $p=\sigma_{i j} \delta_{i j} / 3$ & $c_{\mathrm{n}}=c_{i j} \delta_{i j} / 3$ \\
\hline Deviatoric stress & $q=\sqrt{\frac{3}{2}\left(\sigma_{i j}-p \delta_{i j}\right)\left(\sigma_{i j}-p \delta_{i j}\right)}$ & $c_{\mathrm{s}}=\sqrt{\frac{3}{2}\left(c_{i j}-c_{\mathrm{n}} \delta_{i j}\right)\left(c_{i j}-c_{\mathrm{n}} \delta_{i j}\right)}$ \\
\hline Stress ratio & $\eta=q / p$ & $\chi=c_{\mathrm{s}} / c_{\mathrm{n}}$ \\
\hline Critical stress ratio & $M=\frac{6 \sin \varphi_{\mathrm{c}}}{3-\sin \varphi_{\mathrm{c}}}$ & $F=3 \frac{\left(1+\sin \varphi_{\mathrm{c}}\right)^{\beta}-\left(1-\sin \varphi_{\mathrm{c}}\right)^{\beta}}{\left(1+\sin \varphi_{\mathrm{c}}\right)^{\beta}+2\left(1-\sin \varphi_{\mathrm{c}}\right)^{\beta}}$ \\
\hline
\end{tabular}
the internal frictional angel under triaxial compression conditions.

Tab. 1 Comparison of stress variables in the general stress space and the characteristic stress space

\subsection{Fractional plastic model in characteristic stress space}

Three essential parts for developing a fractional plastic model, including the yield function, the fractional plastic flow rule and the hardening parameter, are all established in the characteristic stress space. Among them, the combination of the proposed fractional plastic flow rule and the 
characteristic stress is used to capture the plastic deformation properties of soils under true 3D stress conditions.

\subsubsection{Yield function}

For the sake of simplicity, the yield function established in the characteristic stress space takes the form of an ellipsoid. It has the same form and convexity with the yield function of the modified Cam-clay (MCC) model (Roscoe and Burland, 1968). The yield function in this paper can be expressed as:

$$
f=c_{\mathrm{s}}^{2}+N^{2}\left(c_{\mathrm{n}}^{2}-c_{\mathrm{n} x} c_{\mathrm{n}}\right)=0
$$

where $N$ is the ratio of the vertical axis and the horizontal axis of the elliptic yield curve as shown in Fig. 5, which is the geometrical role of $M$ in the yield function of the MCC model. The failure stress ratio $F$ is the physical role of $M$ in the MCC model for describing the critical state of soil. $N$ and $F$ equal to $M$ when $\beta=1$. The equivalent consolidation pressure $c_{\mathrm{n} x}$ is the intersection coordinate of the yield curve with the abscissa and can be expressed as Eq. (19). The role of $c_{\mathrm{n} x}$ is to connect the yield curve with the hardening rule, which is described in section 3.2.3.

$$
c_{\mathrm{n} x}=\frac{c_{\mathrm{s}}^{2}+N^{2} c_{\mathrm{n}}^{2}}{N^{2} c_{\mathrm{n}}}
$$

Fig. 5 Yield surface in the meridian plane

Only two stress invariants $c_{\mathrm{n}}$ and $c_{\mathrm{s}}$ are used to construct the yield function. Thus, the yield surface in the $c_{i j}$ space shown in Fig. 6(a) is an axially symmetric ellipsoid with respect to the $c_{\mathrm{n}^{-}}$ axis. However, the corresponding yield surface in the $\sigma_{i j}$ space is an irregular, smooth and convex three-axis symmetric surface when $0<\beta<1$ as shown in Fig. 6(b).

(a)

Fig. 6 Yield surface: (a) in the $c_{i j}$ space; (b) in the $\sigma_{i j}$ space.

Fig. 7 shows the yield curves in the meridian plane and the deviatoric plane of the $\sigma_{i j}$ space. All the yield curves pass through the critical state point $\left(p_{0}, M \cdot p_{0}\right)$. For a certain value of $\beta(0<\beta<1)$, 
yield curve is asymmetric with respect to the $p$-axis in the meridian plane as shown in Fig. 7(a) and is a curved triangle in the deviatoric plane as shown in Fig. 7(b). The value of $\beta$ reflects effects of Lode angle $\theta$ or the intermediate principal stress coefficient $b$ on soil behaviour. The yield curves of $\beta=0.1$ and $\beta=0.01$ are almost coincident, thus $\beta=0.1$ can be taken as the approximate lower bound in this paper for saving the computational cost. As $q$ increases to the critical state along the stress path of constant $p_{0}$ under the monotonic loading, the yield curve of $\beta=0.1$ in the meridian plane gradually evolves and expands, as shown in Fig. 8(a). The yield curve in the deviatoric plane changes from an approximate circle to a curved triangle, as shown in Fig. 8(b), which reflects the stress-induced anisotropy of geomaterials.

(a)

Fig. 7 Yield curves in the $\sigma_{i j}$ space with the decrease of $\beta$ :

(a) in the meridian plane; (b) in the deviatoric plane.

(a)

Fig. 8 Yield curve in the $\sigma_{i j}$ space with the increase of $q$ at a constant $p$ :

(a) in the meridian plane; (b) in the deviatoric plane.

\subsubsection{Fractional plastic flow rule}

The fractional plastic flow rule and the characteristic stress are independent in definition but complementary in property description: one for adjusting the plastic strain increment direction, and the other for describing the soil behaviour under true 3D stress conditions in a simple and unified way. By introducing the new proposed fractional plastic flow rule into the characteristic stress space, a novel sight to describe the soil behaviour is proposed. The fractional plastic flow rule in the characteristic stress space is thus established by referring Eq. (14) as follows:

$$
\mathrm{d} \varepsilon_{i j}^{\mathrm{p}}=\Lambda\left(\frac{\partial^{\mu} f}{\partial c_{\mathrm{n}}{ }^{\mu}} \frac{\partial c_{\mathrm{n}}}{\partial c_{i j}}+\frac{\partial^{\mu} f}{\partial c_{\mathrm{s}}{ }^{\mu}} \frac{\partial c_{\mathrm{s}}}{\partial c_{i j}}\right)
$$

where the fractional partial derivative of the yield function (i.e., Eq. (18)) with respect to $c_{\mathrm{n}}$ and $c_{\mathrm{s}}$ are determined as follows: 


$$
\left\{\begin{array}{l}
\frac{\partial^{\mu} f}{\partial c_{\mathrm{n}}{ }^{\mu}}=\frac{2 N^{2} c_{\mathrm{n}}^{2-\mu}}{\Gamma(3-\mu)}-\frac{N^{2} c_{\mathrm{nx}} c_{\mathrm{n}}^{1-\mu}}{\Gamma(2-\mu)} \\
\frac{\partial^{\mu} f}{\partial c_{\mathrm{s}}{ }^{\mu}}=\frac{2 c_{\mathrm{s}}^{2-\mu}}{\Gamma(3-\mu)}
\end{array}\right.
$$

Introducing $c_{\mathrm{n} x}$ expressed by Eq. (19) and the recursive property of gamma function, i.e., $\Gamma(x+1)=x \cdot \Gamma(x)$ into Eq. (21) yields:

$$
\left\{\begin{array}{l}
\frac{\partial^{\mu} f}{\partial c_{\mathrm{n}}{ }^{\mu}}=\frac{\mu N^{2} c_{\mathrm{n}}^{2}-(2-\mu) c_{\mathrm{s}}^{2}}{\Gamma(3-\mu) c_{\mathrm{n}}^{\mu}} \\
\frac{\partial^{\mu} f}{\partial c_{\mathrm{s}}{ }^{\mu}}=\frac{2 c_{\mathrm{s}}^{2-\mu}}{\Gamma(3-\mu)}
\end{array}\right.
$$

The covariant transformation coefficient, i.e., the partial differential of $c_{\mathrm{n}}$ and $c_{\mathrm{s}}$ with respect to $c_{i j}$, can be derived as follows:

$$
\left\{\begin{array}{l}
\frac{\partial c_{\mathrm{n}}}{\partial c_{i j}}=\frac{1}{3} \delta_{i j} \\
\frac{\partial c_{\mathrm{s}}}{\partial c_{i j}}=\frac{3\left(c_{i j}-c_{\mathrm{n}} \delta_{i j}\right)}{2 c_{\mathrm{s}}}
\end{array}\right.
$$

By substituting Eqs. (22) and (23) into Eq. (20), the plastic strain increment can be expressed as:

$$
\mathrm{d} \varepsilon_{i j}^{\mathrm{p}}=\Lambda\left[\frac{\mu N^{2} c_{\mathrm{n}}^{2}-(2-\mu) c_{\mathrm{s}}^{2}}{\Gamma(3-\mu) c_{\mathrm{n}}^{\mu}} \frac{1}{3} \delta_{i j}+\frac{2 c_{\mathrm{s}}^{2-\mu}}{\Gamma(3-\mu)} \frac{3\left(c_{i j}-c_{\mathrm{n}} \delta_{i j}\right)}{2 c_{\mathrm{s}}}\right]
$$

The ratio of the plastic volumetric strain increment $d \varepsilon_{\mathrm{v}}^{\mathrm{p}}$ to the plastic deviatoric strain increment $\mathrm{d} \varepsilon_{\mathrm{s}}^{\mathrm{p}}$ (i.e., the stress-dilatancy ratio $d=\mathrm{d} \varepsilon_{\mathrm{v}}^{\mathrm{p}} / \mathrm{d} \varepsilon_{\mathrm{s}}^{\mathrm{p}}$ ) can thus be obtained from Eq. (20) together with Eq. (22). The stress-dilatancy ratio $d$ is the reflection of the plastic strain increment direction, which can be expressed as follows.

$$
d=\frac{\mathrm{d} \varepsilon_{\mathrm{v}}^{\mathrm{p}}}{\mathrm{d} \varepsilon_{\mathrm{s}}^{\mathrm{p}}}=\frac{\Lambda \cdot \frac{\partial^{\mu} f}{\partial c_{\mathrm{n}}{ }^{\mu}}}{\Lambda \cdot \frac{\partial^{\mu} f}{\partial c_{\mathrm{s}}{ }^{\mu}}}=\frac{\mu N^{2}-(2-\mu) \chi^{2}}{2 \chi^{2-\mu}}
$$

The condition of $\varphi_{\mathrm{c}}=30^{\circ}$ and $\beta=0.4$ is taken as a special case for demonstration, and $N=0.769$ and $\mu=0.537$ can be determined by methods in the following section 3.3.3. When the soil is loaded from the isotropic compression point $A_{0}$ to the point $A_{1}$ along the conventional triaxial compression path $A_{0} A_{\mathrm{f}}$, the yield curve and the plastic strain increment directions are shown in Fig. 9. As shown in Fig. 9(a), the yield curve in the meridian plane through the current stress point $A_{1}$ is indicated by 
the black ellipsoidal curve. The plastic strain increment directions on the yield curve for $\mu=0.537$ are indicated by the red solid arrow. The normal gradient directions when $\mu=1$ are orthogonal to the yield curve, and is indicated by the blue dash arrow. Since the expression of the yield function is independent of $\theta$, the yield curve in the deviatoric plane is a circle as shown in Fig. 9(b). Therefore, the plastic strain increment direction is perpendicular to the yield circle in the deviatoric plane and independent of $\mu$. The overview of the yield surface and the plastic strain increment directions in the $c_{i j}$ space are shown in Fig. 10.

(a)

Fig. 9 Plastic strain increment direction in the $c_{i j}$ space at the current loading point:

(a) in the meridian plane with $\theta=\theta_{A}$; (b) in the deviatoric plane with $p=p_{A}$.

Fig. 10 Plastic strain increment direction in the $c_{i j}$ space.

For the condition of $\mu=0.537$, the variation rules of the plastic strain increments with increasing $\chi$ are shown in Fig. 11. When $\chi=0$, the plastic strain increment direction is horizontal to the right and is independent of $\mu$, which means that plastic deviatoric strain does not occur under the isotropic compression conditions, i.e., $\mathrm{d} \varepsilon_{\mathrm{s}}^{\mathrm{p}}=0$. With the increase of $\chi$, the plastic strain increment directions gradually change from horizontal to vertical, and $\mathrm{d} \varepsilon_{\mathrm{v}}^{\mathrm{p}}>0$. When $\chi$ reaches $F$, the stress state reaches the critical state, but not the peak of the yield curve. The plastic strain increment direction is vertically upward, which means that plastic volumetric strain does not occur, i.e., $\mathrm{d} \varepsilon_{\mathrm{v}}^{\mathrm{p}}=0$. It should be noticed that for a fixed value of $\chi$, the plastic strain increment directions on different yield curves are the same, as shown in Fig. 11.

Fig. 11 Plastic strain increment direction during the loading process.

The stress-dilatancy relationship actually reflects the variation rules of the plastic strain increment direction. The stress-dilatancy relationship $d-\chi$ at different values of $\beta$ is shown in Fig. 12. $\varphi_{\mathrm{c}}=30^{\circ}$ is used for demonstration. The model parameters $N$ and $\mu$ correspond to $\beta=0.1,0.4,0.7$ 
and 1.0 are listed in Tab. 2. Once the material parameters $\varphi_{\mathrm{c}}$ and $\beta$ are obtained, the one-to-one model parameters $N$ and $\mu$ can be determined by methods in the following section 3.3.3. For $\beta=1, N$ and $F$ equal to $M, \mu$ degenerates to 1 and the $d-\chi$ relationship is the same with the stress-dilatancy relationship of MCC model. With the decrease of $\beta$, the determined values of $N$ and $\mu$ decrease accordingly as listed in Tab. 2 , and the $d-\chi$ relationship curves decline with the decreasing $\beta$. Different levels of stress-dilatancy under compression and extension conditions can be reflected as shown in Fig. 12(b), which benefits from the characteristic stress. The difference degree of the stress-dilatancy under triaxial compression and extension conditions enlarges with the decreasing $\beta$. Fig. 13 shows the stress-dilatancy curves in the characteristic stress space and the general stress space for $\beta=0.1$. Stress-dilatancy curves at $b=0,0.25,0.5,0.75$ and 1 in the characteristic stress space are coincident, as shown in Fig. 13(a). These coincident curves in the $c_{i j}$ space correspond to a series of stress-dilatancy curves in the $\sigma_{i j}$ space, as shown in Fig. 13(b).

Tab. 2 Parameters in the characteristic stress spaces

\begin{tabular}{cccc}
\hline$\varphi_{\mathrm{c}}$ & $\beta$ & $N$ & $\mu$ \\
\hline & 1.0 & 1.200 & 1.000 \\
$30^{\circ}$ & 0.7 & 1.016 & 0.807 \\
& 0.4 & 0.769 & 0.537 \\
& 0.1 & 0.381 & 0.158 \\
\hline
\end{tabular}

(a)

(b)

Fig. 12 Stress-dilatancy relationship for different $\beta$-values: (a) in the $c_{i j}$ space; (b) in the $\sigma_{i j}$ space.

(a)

Fig. 13 Stress-dilatancy curves when $\beta=0.1$ : (a) in the $c_{i j}$ space; (b) in the $\sigma_{i j}$ space.

\subsubsection{Hardening parameter}

Hardening parameter can be used to describe the hardening/softening rule as well as the evolution of the yield surface. Some valuable studies on hardening parameter have been done to describe the strain hardening and softening properties (Yao et al., 2009; Wang et al., 2018), the stress-dilatancy and state-dependency (Li and Dafalias, 2000) and the particle breakage effect (Xiao and Liu, 2017) et al.. Similar with the MCC model, the plastic volumetric strain is chosen as the hardening parameter in this paper, which can be derived from the linear relationship between $e$ and 
$\ln p$ for normally consolidated clay under isotropic compression conditions $\left(\sigma_{1}=\sigma_{2}=\sigma_{3}\right)$. As shown in Fig. 14, the relationship between the void ratio $e$ and $\ln c_{\mathrm{n}}$ can be easily obtained from the linear relationship of $e \sim \ln p$ under the isotropic compression conditions. Corresponding to the compression index $\lambda$ and the swelling index $\kappa$ in the general stress space, the compression index and the swelling index in the characteristic stress space become $\lambda / \beta$ and $\kappa / \beta$.

Fig. $14 e-\ln c_{\mathrm{n}}$ relationship in the $c_{i j}$ space.

The way to derive the relationship between the plastic volumetric strain $\varepsilon_{\mathrm{v}}^{\mathrm{p}}$ and the equivalent consolidation pressure $c_{\mathrm{n} x}$ in the $c_{i j}$ space is listed in Tab. 3. The equivalent consolidation pressure can be written as follows:

$$
c_{\mathrm{n} x}=c_{\mathrm{n} 0} \exp \left(\frac{\varepsilon_{\mathrm{v}}^{\mathrm{p}}}{\rho_{\mathrm{p}}}\right)
$$

where $\rho_{\mathrm{p}}=(\lambda-\kappa) / \beta /\left(1+e_{0}\right) . e_{0}$ is the initial void ratio. $c_{\mathrm{n} 0}$ is the initial mean characteristic stress.

Tab. 3 Rule of the strain hardening for clay in the characteristic stress space

Characteristic stress space

Initial mean characteristic stress

$$
c_{\mathrm{n} 0}=p_{0}^{\beta}
$$

Void ratio $e$

$$
\Delta e=e-e_{0}=-\frac{\lambda}{\beta} \ln \frac{c_{\mathrm{n} x}}{c_{\mathrm{n} 0}}
$$

volumetric strain $\varepsilon_{\mathrm{V}}$

$$
\varepsilon_{\mathrm{v}}=\frac{-\Delta e}{1+e_{0}}=\frac{\lambda}{\beta\left(1+e_{0}\right)} \ln \frac{c_{\mathrm{n} x}}{c_{n 0}}
$$

Elastic volumetric strain $\varepsilon_{\mathrm{V}}{ }^{\mathrm{e}}$

$$
\varepsilon_{\mathrm{v}}^{\mathrm{e}}=\frac{-\Delta e^{\mathrm{e}}}{1+e_{0}}=\frac{\kappa}{\beta\left(1+e_{0}\right)} \ln \frac{c_{\mathrm{n} x}}{c_{\mathrm{n} 0}}
$$

Plastic volumetric strain $\varepsilon_{\mathrm{V}}{ }^{\mathrm{p}}$

$$
\varepsilon_{\mathrm{v}}^{\mathrm{p}}=\varepsilon_{\mathrm{v}}-\varepsilon_{\mathrm{v}}^{\mathrm{e}}=\frac{\lambda-\kappa}{\beta\left(1+e_{0}\right)} \ln \frac{c_{\mathrm{n} x}}{c_{\mathrm{n} 0}}
$$

$$
\text { Equivalent consolidation pressure } c_{\mathrm{n} x} \quad c_{\mathrm{n} x}=c_{\mathrm{n} 0} \exp \left(\beta \frac{1+e_{0}}{\lambda-\kappa} \varepsilon_{\mathrm{v}}^{\mathrm{p}}\right)=c_{\mathrm{n} 0} \exp \left(\frac{\varepsilon_{\mathrm{v}}^{\mathrm{p}}}{\rho_{\mathrm{p}}}\right)
$$

\subsection{Stress-strain relationship}

The stress-strain relationship of the normally consolidated clay can be obtained in the $c_{i j}$ space. 
In the elastoplastic theory framework, the total strain increment $\mathrm{d} \varepsilon_{i j}$ is divided into the elastic strain increment $\mathrm{d} \varepsilon_{i j}^{\mathrm{e}}$ and the plastic strain increment $\mathrm{d} \varepsilon_{i j}^{\mathrm{p}}$ as follows:

$$
\mathrm{d} \varepsilon_{i j}=\mathrm{d} \varepsilon_{i j}^{\mathrm{e}}+\mathrm{d} \varepsilon_{i j}^{\mathrm{p}}
$$

\subsubsection{Elastic strain increment}

The elastic strain increment can be calculated by the Hook's law.

$$
\mathrm{d} \varepsilon_{i j}^{\mathrm{e}}=\frac{1+v}{E} \mathrm{~d} \sigma_{i j}-\frac{v}{E} \mathrm{~d} \sigma_{k k} \delta_{i j}
$$

Based on Eq. (28) and Eq. (16), the elastic principal strain can also be expressed by the characteristic principal stresses as follows:

$$
\begin{aligned}
& \mathrm{d} \varepsilon_{1}^{\mathrm{e}}=\frac{1}{\beta E} c_{1}^{\frac{1}{\beta}-1} \mathrm{~d} c_{1}-\frac{v}{\beta E}\left(c_{2}^{\frac{1}{\beta}-1} \mathrm{~d} c_{2}+c_{3}^{\frac{1}{\beta}-1} \mathrm{~d} c_{3}\right) \\
& \mathrm{d} \varepsilon_{2}^{\mathrm{e}}=\frac{1}{\beta E} c_{2}^{\frac{1}{\beta}-1} \mathrm{~d} c_{2}-\frac{v}{\beta E}\left(c_{3}^{\frac{1}{\beta}-1} \mathrm{~d} c_{3}+c_{1}^{\frac{1}{\beta}-1} \mathrm{~d} c_{1}\right) \\
& \mathrm{d} \varepsilon_{3}^{\mathrm{e}}=\frac{1}{\beta E} c_{3}^{\frac{1}{\beta}-1} \mathrm{~d} c_{3}-\frac{v}{\beta E}\left(c_{1}^{\frac{1}{\beta}-1} \mathrm{~d} c_{1}+c_{2}^{\frac{1}{\beta}-1} \mathrm{~d} c_{2}\right)
\end{aligned}
$$

where $v$ is the Poisson's ratio. The Young's elastic modulus $E$ can be expressed as the function of the mean characteristic stress, as follows:

$$
E=3(1-2 v) \frac{1+e_{0}}{\kappa} c_{\mathrm{n}}^{\frac{1}{\beta}}
$$

\subsubsection{Plastic strain increment}

The consistency condition, i.e., the total differential of the yield function Eq. (18), can be expressed as follows:

$$
\mathrm{d} f=\frac{\partial f}{\partial c_{\mathrm{n}}} \mathrm{d} c_{\mathrm{n}}+\frac{\partial f}{\partial c_{\mathrm{s}}} \mathrm{d} c_{s}+\frac{\partial f}{\partial c_{\mathrm{n} x}} \frac{\partial c_{\mathrm{n} x}}{\partial \varepsilon_{\mathrm{v}}^{\mathrm{p}}} \mathrm{d} \varepsilon_{\mathrm{v}}^{\mathrm{p}}=0
$$

where $\partial f / \partial c_{\mathrm{n}}=N^{2}\left(2 c_{\mathrm{n}}-c_{\mathrm{n} x}\right), \partial f / \partial c_{\mathrm{s}}=2 c_{\mathrm{s}}, \partial f / \partial c_{\mathrm{n} x}=-N^{2} c_{\mathrm{n}}$. The derivative of $c_{\mathrm{n} x}$ with respect to $\varepsilon_{\mathrm{v}}^{\mathrm{p}}$ can derived from Eq. (26):

$$
\frac{\partial c_{\mathrm{n} x}}{\partial \varepsilon_{\mathrm{v}}^{\mathrm{p}}}=\frac{c_{\mathrm{n} x}}{\rho_{\mathrm{p}}}
$$

By substituting $d \varepsilon_{\mathrm{v}}^{\mathrm{p}}$ derived from Eq. (24) together with Eq. (32) into Eq. (31), the plastic multiplier $\Lambda$ can be derived as follows: 


$$
\Lambda=\rho_{\mathrm{p}} \frac{\Gamma(3-\mu) c_{\mathrm{n}}^{\mu}\left[N^{2}\left(2 c_{\mathrm{n}}-c_{\mathrm{nx}}\right) \mathrm{d} c_{\mathrm{n}}+2 c_{\mathrm{s}} \mathrm{d} c_{\mathrm{s}}\right]}{N^{2} c_{\mathrm{n}} c_{\mathrm{n} x}\left[\mu N^{2} c_{\mathrm{n}}^{2}-(2-\mu) c_{\mathrm{s}}^{2}\right]}
$$

The plastic strain increment is derived by substituting the plastic multiplier $\Lambda$ in Eq. (33) together with $c_{\mathrm{nx}}$ in Eq. (19) into Eq. (24).

$$
\mathrm{d} \varepsilon_{i j}^{\mathrm{p}}=\rho_{\mathrm{p}}\left[\frac{\delta_{i j}}{3}+\frac{3 c_{\mathrm{n}}^{\mu} c_{\mathrm{s}}^{1-\mu}\left(c_{i j}-c_{\mathrm{n}} \delta_{i j}\right)}{\mu N^{2} c_{\mathrm{n}}^{2}-(2-\mu) c_{\mathrm{s}}^{2}}\right] \frac{\left(N^{2} c_{\mathrm{n}}^{2}-c_{\mathrm{s}}^{2}\right) \mathrm{d} c_{\mathrm{n}}+2 c_{\mathrm{n}} c_{\mathrm{s}} \mathrm{d} c_{\mathrm{s}}}{c_{\mathrm{n}}\left(N^{2} c_{\mathrm{n}}^{2}+c_{\mathrm{s}}^{2}\right)}
$$

The stress-strain relationship in Eq. (34) is totally expressed by the characteristic stresses. It can also degenerate into the MCC model when $\beta=1$. The parameters in Eq. (34) can be determined by the traditional triaxial tests.

\subsubsection{Parameters in the proposed model}

There are only five independent material parameters included in the proposed fractional elastoplastic model, including $\lambda, \kappa, v, \varphi_{\mathrm{c}}$ and $\beta$, each of which has clear physical significance. Among them, $\lambda, \kappa, v$ and $\varphi_{\mathrm{c}}$ are the same as the material parameters included in the MCC model. Only one material parameter $\beta$ is added, which can be determined by combining $\varphi_{\mathrm{c}}$ for $b=0$ with an additional internal friction angle $\varphi_{b}$ for $b \neq 0$. According to the critical stress ratio $F$ in the $c_{i j}$ space, which is independent of the $b$-value, the equation $\left.F\right|_{b=0}=\left.F\right|_{b}$ can be used to determine $\beta$.

$$
\frac{\sqrt{2}\left(R_{\mathrm{c}}^{\beta}-1\right)}{R_{\mathrm{c}}^{\beta}+2}=\frac{\sqrt{\left[R_{b}^{\beta}-\left(b R_{b}-b+1\right)^{\beta}\right]^{2}+\left[\left(b R_{b}-b+1\right)^{\beta}-1\right]^{2}+\left(R_{b}^{\beta}-1\right)^{2}}}{\left(b R_{b}-b+1\right)^{\beta}+R_{b}^{\beta}+1}
$$

where $R_{\mathrm{c}}=\left(1+\sin \varphi_{\mathrm{c}}\right) /\left(1-\sin \varphi_{\mathrm{c}}\right)$ and $R_{b}=\left(1+\sin \varphi_{b}\right) /\left(1-\sin \varphi_{b}\right)$. Especially, $\beta$ can be determined with the internal friction angle $\varphi_{\mathrm{e}}$ for $b=1$ by the following equation (Lu et al., 2017).

$$
\frac{\left(1+\sin \varphi_{\mathrm{c}}\right)^{\beta}-\left(1-\sin \varphi_{\mathrm{c}}\right)^{\beta}}{\left(1+\sin \varphi_{\mathrm{c}}\right)^{\beta}+2\left(1-\sin \varphi_{\mathrm{c}}\right)^{\beta}}=\frac{\left(1+\sin \varphi_{\mathrm{e}}\right)^{\beta}-\left(1-\sin \varphi_{\mathrm{e}}\right)^{\beta}}{2\left(1+\sin \varphi_{\mathrm{e}}\right)^{\beta}+\left(1-\sin \varphi_{\mathrm{e}}\right)^{\beta}}
$$

Thus, $\beta$ actually reflects the proportional relation of $\varphi_{\mathrm{c}}$ and $\varphi_{\mathrm{e}}$.

In addition, two model parameters, i.e., $N$ and $\mu$, are include in the proposed model, which can be determined by material parameters without the need for additional tests. The determined methods of the model parameters are given as follows:

Under the triaxial compression condition with $p=$ constant, $\varepsilon_{\mathrm{v}}^{\mathrm{e}}=0$. Therefore, $\varepsilon_{\mathrm{v}}=\varepsilon_{\mathrm{v}}^{\mathrm{p}}$. When the soil is loaded to the critical state, the variation of the void ratio equals to the vertical distance between the NCL and the CSL, and can be expressed as $\ln 2 \cdot(\lambda-\kappa)(\mathrm{Yu}, 1998)$. Thus, $\varepsilon_{\mathrm{v}}^{\mathrm{p}}$ can be 
calculated by the following equation:

$$
\varepsilon_{\mathrm{v}}^{\mathrm{p}}=\frac{e_{0}-e}{1+e_{0}}=\frac{\ln 2 \cdot(\lambda-\kappa)}{1+e_{0}}
$$

Therefore, $c_{\mathrm{n} x}$ can be derived by substituting Eq. (37) into Eq. (26):

$$
c_{\mathrm{n} x}=c_{\mathrm{n} 0} \exp \left(\varepsilon_{\mathrm{v}}^{\mathrm{p}} / \rho_{\mathrm{p}}\right)=2^{\beta} c_{\mathrm{n} 0}
$$

The critical principal stress $\sigma_{1 \mathrm{z}}=p_{0}(1+2 M / 3)$ and $\sigma_{3 \mathrm{z}}=p_{0}(1-M / 3)$. The critical mean characteristic stress $c_{\mathrm{n} z}=\left(\sigma_{1 z}^{\beta}+2 \sigma_{3 z}^{\beta}\right) / 3$ is thus obtained. By substituting the critical stress state $\left(c_{\mathrm{nz}}, c_{\mathrm{sz}}\right)=\left(c_{\mathrm{nz}}, F \cdot c_{\mathrm{nz}}\right)$ into Eq. (19), the equivalent consolidation pressure is:

$$
c_{\mathrm{n} x}=c_{\mathrm{nz}}\left(F^{2} / N^{2}+1\right)
$$

Combining Eq. (38) with (39), one can get $N$ as follows:

$$
N=\sqrt{\frac{F^{2} c_{\mathrm{nz}}}{2^{\beta} c_{\mathrm{n} 0}-c_{\mathrm{nz}}}}=\sqrt{\frac{F^{2}\left[(1+2 M / 3)^{\beta}+2(1-M / 3)^{\beta}\right]}{3 \cdot 2^{\beta}-\left[(1+2 M / 3)^{\beta}+2(1-M / 3)^{\beta}\right]}}
$$

where $F$ can be calculated by $\varphi_{\mathrm{c}}$ and $\beta$, and $M$ is the function of $\varphi_{\mathrm{c}}$, as listed in Tab. 1 . Therefore, $N$ is actually determined only by $\varphi_{\mathrm{c}}$ and $\beta$.

When the critical state condition is reached, $\chi$ reaches $F$, and $d$ in Eq. (25) equals 0 . The fractional order $\mu$ can thus be determined by Eq. (25) as follows:

$$
\mu=\frac{2 F^{2}}{N^{2}-F^{2}}
$$

The fractional order $\mu$ is also determined by two material parameters, $\varphi_{\mathrm{c}}$ and $\beta$.

\subsection{Critical state}

The ultimate condition that plastic shearing continues indefinitely without changes in volume and effective stresses has been known as the critical state (Muir Wood, 1990). It has been testified that the critical state is closely related to the true $3 \mathrm{D}$ stress conditions and should be reflected in constitutive models (Zhao and Guo, 2013; Xiao and Liu, 2017; Zhou et al., 2017), which can be reflected by the characteristic stress in the proposed fractional elastoplastic model.

The soil parameters for Fujinomori clay, which are stated in section 4.1, are used to exhibit the critical state properties that reflected by the proposed model. The stress paths of $b=0, b=0.5$ and $b=1$ when $p=$ constant under the drained condition and the undrained condition $\left(\varepsilon_{\mathrm{V}}=0\right)$ are shown in the $c_{i j}$ space in Fig. 15(a) and the $\sigma_{i j}$ space in Fig. 15(b), respectively. Stress paths of $b=0, b=0.5$ and 
$b=1$ in the $c_{i j}$ space are different but reach to a unique critical state line, which correspond to different stress paths and different CSLs in the $\sigma_{i j}$ space. That is, the critical state is reached when $\chi$ equals to $F$. At the same time, $\eta$ reaches the critical stress ratio $M_{b}$ for a certain value of $b$ in the $\sigma_{i j}$ space. The critical state behaviour of soils under true 3D stress conditions is thus reflected by the unique critical state line in the $c_{i j}$ space.

Fig. 15 Stress paths and critical state lines: (a) in the $c_{i j}$ space; (b) in the $\sigma_{i j}$ space.

\section{Experimental verification}

The existing fractional models for soil cannot describe the deformation properties of soils under the true $3 \mathrm{D}$ stress conditions. The capability of the proposed fractional elastoplastic model in capturing the deformation properties of soil is thus examined by predicting test results and comparing with the predictions of the MCC model. The true triaxial test results of the new Fujinomori (NF) clay under the drained conditions (Chowdhury and Nakai, 1998) and the Grundite clay under the undrained conditions (Lade and Musante, 1978) are used in this paper.

\subsection{Fujinomori clay}

Drained true triaxial tests were performed on the normally consolidated NF clay at a constant mean stress $p=196 \mathrm{kPa}$. The values of the intermediate principal stress coefficient $b$ were set as 0 , $0.268,0.5$ and 0.732 , corresponding to different stress Lode angles $\theta=0^{\circ}, 15^{\circ}, 30^{\circ}, 45^{\circ}$, respectively. The values of material parameters for the NF clay used in the models are $\varphi_{\mathrm{c}}=33.7^{\circ}, \lambda /\left(1+e_{0}\right)=0.0508$, $\kappa /\left(1+e_{0}\right)=0.0112$ and $\nu=0.3$. The characteristic material parameter $\beta=0.1$ is used for predictions. Accordingly, the model parameters $N=0.4248$ and $\mu=0.1654$ are determined by Eqs. (40) and (41).

The experimental data from Chowdhury and Nakai (1998) are arranged in terms of the relation among principal stress ratio $\sigma_{1} / \sigma_{3}$, volumetric strain $\varepsilon_{\mathrm{v}}$ and principal strains $\left(\varepsilon_{1}, \varepsilon_{2}\right.$ and $\left.\varepsilon_{3}\right)$, as indicated by the open circles in Fig. 16. These true triaxial test data at different $b$-values are predicted by the proposed 3D fractional elastoplastic model and the MCC model, which are indicated by the red solid line and the black dash line, respectively. Both the proposed model and the MCC model can well predict the stress-strain behaviour under triaxial compressions conditions, i.e., $b=0$, as shown in Fig. 16(a). However, for $b=0.268,0.5$ and 0.732 , the experimental stress-strain behaviour 
is predicted by the proposed fractional model better than the MCC model, as shown in Fig. 16(b) (d). That is, the proposed fractional elastoplastic model can well capture the strength property and the deformation behaviour of the normally consolidated NF clay under the drained triaxial conditions.

(a)

(b) (c)

(d)

Fig. 16 Comparison between test results (data from Chowdhury and Nakai, 1998) and predicted results under true $3 \mathrm{D}$ stress conditions at $p=196 \mathrm{kPa}$ with: (a) $b=0$; (b) $b=0.268$; (c) $b=0.5$; (d) $b=0.732$.

The relationship between the strains under true 3D stress conditions is actually a reflection of the deformation flow direction of soil. Test results with different $b$-values are arranged in terms of the relation between $\varepsilon_{2}$ and $\varepsilon_{1}$ and the relation between $\varepsilon_{3}$ and $\varepsilon_{1}$, as shown by dots in Fig. 17 . Test results shown in Fig. 17(a) indicate that $\varepsilon_{2}$ is negative, i.e., extensive, when $b=0$ and $b=0.268$, and is positive, i.e., compressive, when $b=0.5$ and $b=0.732$. Fig. $17(\mathrm{~b})$ shows that $\varepsilon_{3}$ is extensive in all the cases, and the extent of extensive gradually decreases for higher $b$-values. The experimental relation between $\varepsilon_{2}$ and $\varepsilon_{1}$ and between $\varepsilon_{3}$ and $\varepsilon_{1}$ for a constant $b$-value, as well as the variation rule for different $b$-values, are all reasonably captured by the proposed fractional model better than the MCC model.

(a)

(b)

Fig. 17 Comparisons between test results (data from Chowdhury and Nakai, 1998) and predicted results between principal stains: (a) $\varepsilon_{2}-\varepsilon_{1}$; (a) $\varepsilon_{3}-\varepsilon_{1}$.

\subsection{Grundite clay}

Undrained true triaxial experimental study was performed by Lade and Musante (1978) to study the influence of $b$ on the stress-strain behaviour, pore pressure and strength properties of normally consolidated Grundite clay. Cubical specimens of the remolded Grundite clay were tested at a consolidation pressure of $\sigma_{\mathrm{c}}=147 \mathrm{kPa}$ and all three principal stresses are independently controlled. $b$ was maintained constant in each test, and $b=0.00,0.21,0.40,0.70$ and 0.95 , separately. Soil parameters for predictions are from Yao et al. (2004) and listed as: $\varphi_{\mathrm{c}}=28.2^{\circ}, \lambda /\left(1+e_{0}\right)=0.0846$, $\kappa /\left(1+e_{0}\right)=0.0169$ and $v=0.27 . \varphi_{\mathrm{e}}=31.18^{\circ}$ from the test with $b=0.95$ is used to determine $\beta=0.303$. 
Correspondingly, the model parameters $N=0.6289$ and $\mu=0.4222$ are determined from Eqs. (40) and (41).

Fig. 18 shows the comparison between test data and predicted lines presented in terms of relations among the principal stress ratio $\sigma_{1} / \sigma_{3}$, the pore pressure ratio $u / \sigma_{\mathrm{c}}$ and the principal strain $\varepsilon_{1}$, separately. When $b=0$, the predicted lines of the proposed fractional elastoplastic model are almost the same with the predicted lines of the MCC model, and they both capture the experimental stress-strain behaviour well, as shown in Fig. 18(a). The shear strengths of the Grundite clay with $b=0.21,0.40,0.70$ and 0.95 are well captured by the proposed model, but obviously overpredicted by the MCC model, as shown in Fig. 18(b)-(d). In addition, the proposed fractional elastoplastic model better predicts the relationship between $u / \sigma_{\mathrm{c}}$ and $\varepsilon_{1}$ for $b \neq 0$ than the MCC model.

(a)

(b) (c) (d) (e)

Fig. 18 Comparisons between test results (data from Lade and Musante, 1978) and predicted results

$$
\text { with } \sigma_{\mathrm{c}}=147 \mathrm{kPa} \text { and: (a) } b=0 \text {; (b) } b=0.21 \text {; (b) } b=0.40 \text {; (d) } b=0.70 \text {; (e) } b=0.95 \text {. }
$$

The test data between principal strains under undrained conditions are compared with the predicted lines of the proposed fractional elastoplastic model and the MCC model. Test results are represented by dots. The predictions of the proposed model and the MCC model are represented by the red solid lines and the black dash lines, respectively. The test results and the predictions of $\varepsilon_{2}$ and $\varepsilon_{3}$ are plotted versus $\varepsilon_{1}$ for different $b$-values, as shown in Fig. 19. As shown in Fig. 19(a), $\varepsilon_{2}$ changes from extension to compression as $b$ increases. $\varepsilon_{3}$ is under extension for all $b$-values, and the extent of extension gradually decreases with a larger $b$-value, as shown in Fig. 19(b). By comparison, the ability of the proposed model to capture the relationship between the principal strains under true 3D stress conditions is demonstrated to be better than the MCC model.

(a)

(b)

Fig. 19 Comparisons between test results (data from Lade and Musante, 1978) and predicted results

$$
\text { between principal stains: (a) } \varepsilon_{2}-\varepsilon_{1} \text {; (b) } \varepsilon_{3}-\varepsilon_{1} \text {. }
$$


In the present study, a novel 3D fractional plastic flow rule without limiting the coordinate

575 basis of the differentiable function was proposed, which is in coordination with the framework of the elastoplastic theory. The plastic strain increment direction for soil under the true 3D stress conditions can be determined in two steps: firstly, determine the direction in an interim coordinate system, and then transform it into general stress space. The 3D fractional plastic flow rule offers a possibility to develop a fractional elastoplastic model for geomaterials, like soils, concretes and rocks, even for the hydrostatic pressure independent materials, like metallic materials.

In the characteristic stress space, by combining the proposed fractional plastic flow rule with the ellipsoidal yield function, and further taking the plastic volumetric strain as the hardening parameter, a 3D fractional elastoplastic constitutive model for soil was proposed for the first time. The proposed model has only one more material parameter, i.e. $\beta$, than the MCC model. It can well reflect the variation rule of the plastic strain increment direction under complex loading conditions and the properties of the critical state for soil. The comparison between the model predictions and the test results indicates that the proposed model can properly capture the strength and deformation behaviour of soils under true $3 \mathrm{D}$ stress conditions.

\section{Acknowledgements}

This work was supported by the National Key Research and Development Program of China (Grant No. 2016YFC0701104), the National Natural Science Foundation of China (Grant Nos. 51522802, 51778026, 51421005 and 51538001) and the National Natural Science Foundation of Beijing (8161001).

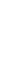


$N$ ratio of the vertical axis and the horizontal axis of the yield curve

$Q_{i j} \quad$ orthogonal tensor for transformation

$R_{b} \quad$ principal stress ratio under constant $b$

$R_{\mathrm{c}} \quad$ principal stress ratio under triaxial compression conditions

$S_{k l}, S_{k} \quad$ interim stress tensor and its eigenvalue

$I_{1}, I_{2}, I_{3} \quad$ first, second and third stress invariants

$b \quad$ intermediate principal stress coefficient

$c_{i j} \quad$ characteristic stress tensor

$c_{i} \quad$ characteristic principal stress

$c_{\mathrm{n}}, c_{\mathrm{s}} \quad$ mean characteristic stress and characteristic deviatoric stress

$c_{\mathrm{n} 0} \quad$ initial mean characteristic stress

d stress-dilatancy ratio

$e, e_{0} \quad$ void ratio and its initial value

$l_{i}^{j} \quad$ covariant transformation coefficient

$p, q, \theta \quad$ mean stress, deviatoric stress and stress Lode angle

$p_{0} \quad$ initial mean stress

$p_{\mathrm{r}} \quad$ reference stress for dimensionless transformation

$m_{i j}^{(\sigma)}, m_{k l}^{(S)} \quad$ components of the fractional gradient vector of $f$ in the $\sigma_{i j}$ space and in the $S_{k l}$ space

$n_{i j}^{(\sigma)}, n_{k l}^{(S)} \quad$ components of the gradient vector of $g$ in the $\sigma_{i j}$ space and in the $S_{k l}$ space

$\mathbf{m}_{\mathbf{m} \mu}, \mathbf{m}_{\mathbf{d} \mu} \quad$ components of the fractional gradient vector of $f$

$\mathbf{n}_{\mathbf{m} g}, \mathbf{n}_{\mathbf{d} g} \quad$ components of the gradient vector of $g$

$\Gamma(\cdot) \quad$ Euler Gamma function

1 plastic multiplier for fractional plastic flow rule

$\mathrm{d} \lambda$ plastic multiplier for classical plastic flow rule 
$\beta \quad$ characteristic stress index

$\delta_{i j} \quad$ Kronker delta tensor

$\varepsilon_{i j}, \varepsilon_{i j}^{\mathrm{e}}, \varepsilon_{i j}^{\mathrm{p}} \quad$ strain tensor, elastic strain tensor and plastic strain tensor

$\varepsilon_{\mathrm{v}}, \varepsilon_{\mathrm{v}}^{\mathrm{e}}, \varepsilon_{\mathrm{v}}^{\mathrm{p}} \quad$ volumetric strain, elastic volumetric strain and plastic volumetric strain

$\varepsilon_{\mathrm{s}}^{\mathrm{p}} \quad$ deviatoric plastic strain related to $q$

$\varepsilon_{\theta}^{\mathrm{p}} \quad$ deviatoric plastic strain related to $\theta$

$\eta \quad$ stress ratio

$\kappa \quad$ swelling index

$\lambda \quad$ compression index

$\mu \quad$ fractional order

$v \quad$ Poisson's ratio

$\sigma_{i} \quad$ principal stress

$\sigma_{i j} \quad$ stress tensor

$\varphi \quad$ internal friction angle

$\chi \quad$ characteristic stress ratio

600 

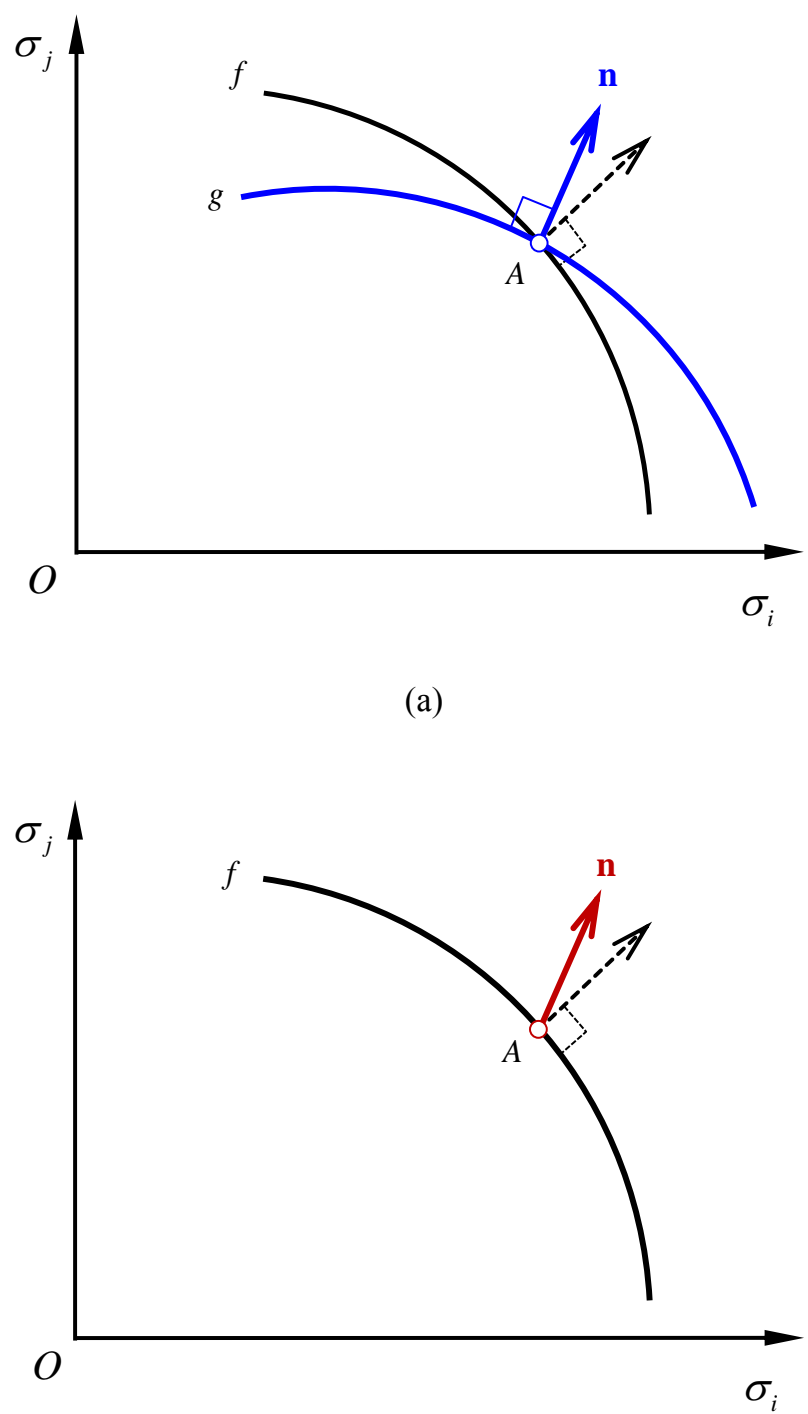

(b)

Fig. 1 Two ways to determine the plastic flow direction:

(a) orthogonal way; (b) non-orthogonal way. 


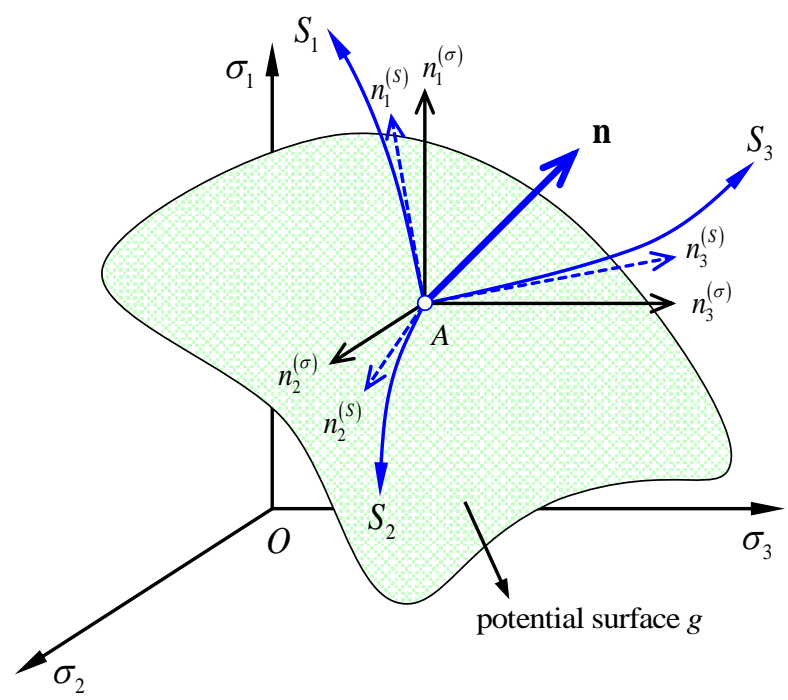

Fig. 2 Coordinate transformation of the plastic flow direction. 

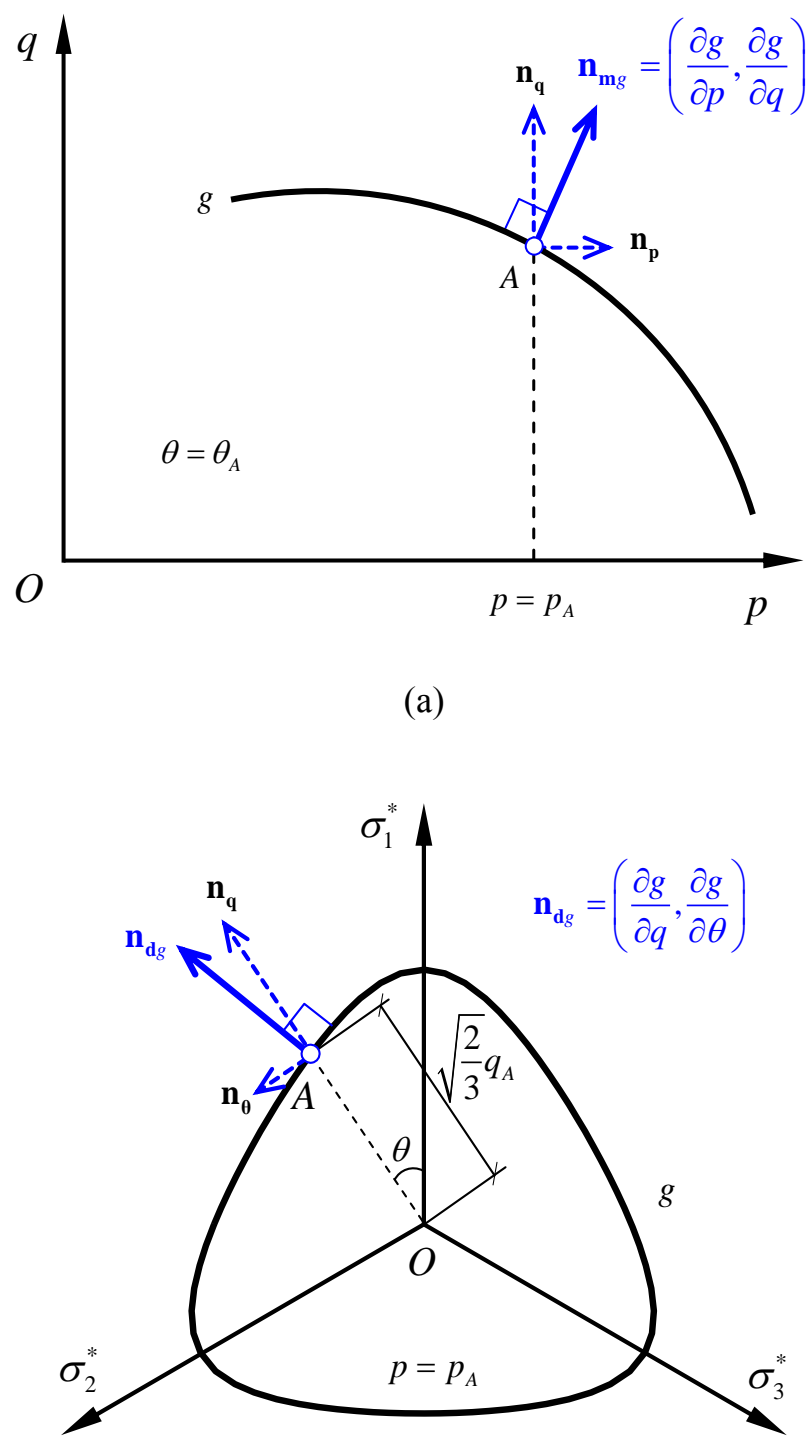

Fig. 3 Plastic flow direction in: (a) the meridian plane with $\theta=\theta_{A}$; (b) the deviatoric plane with $p=p_{A}$. 

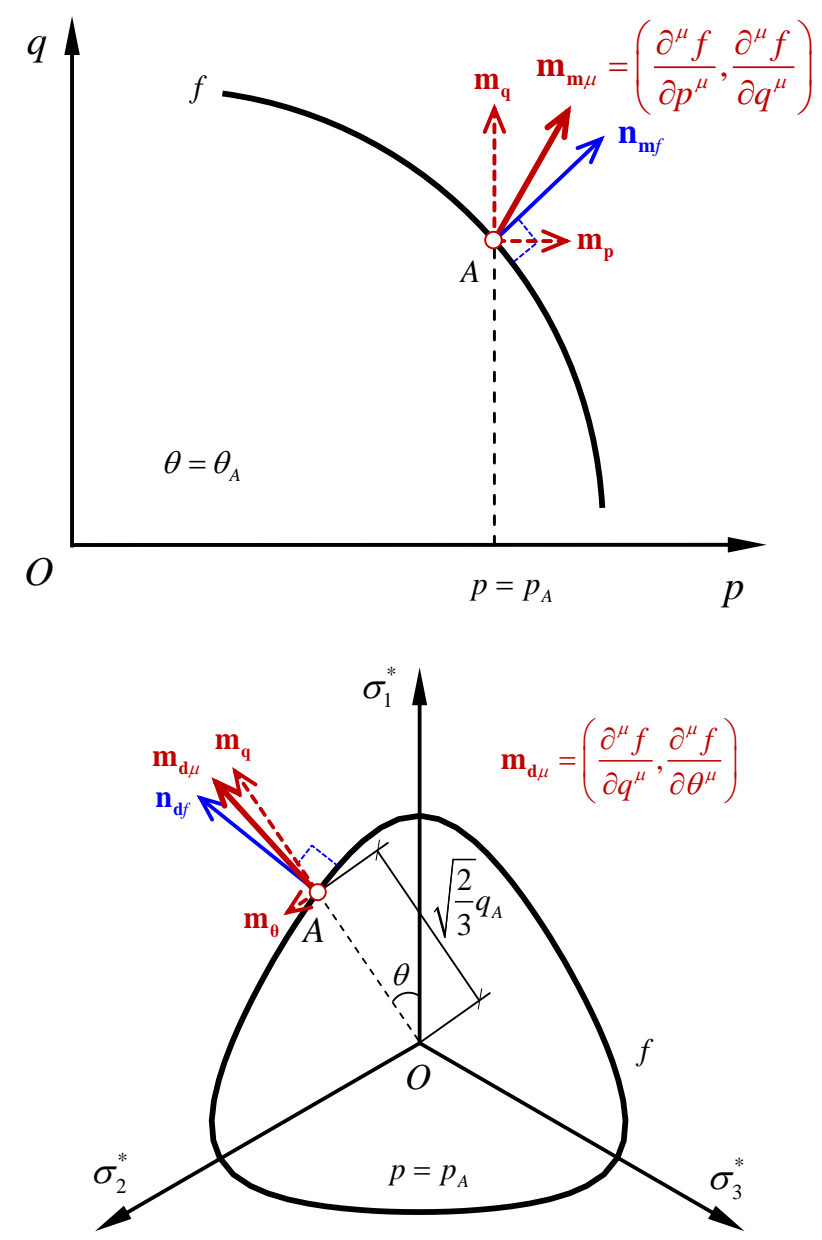

(a)

(b)

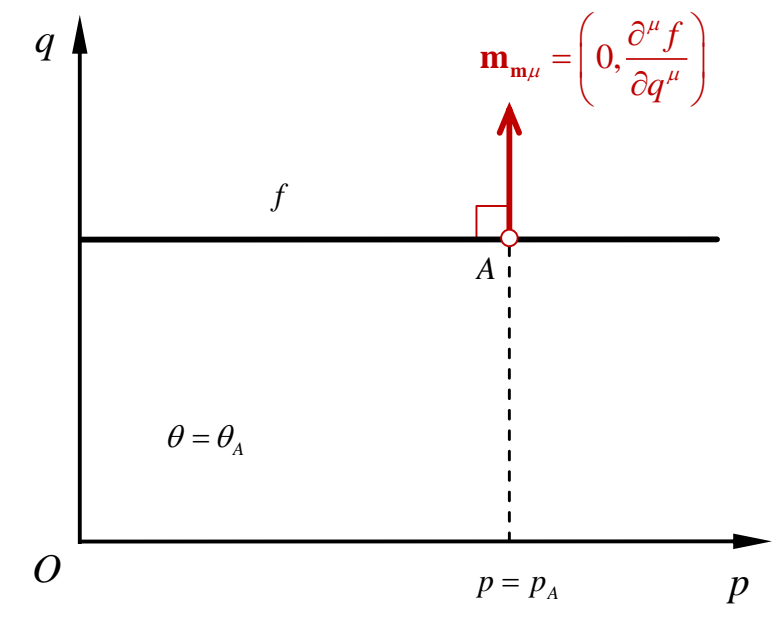




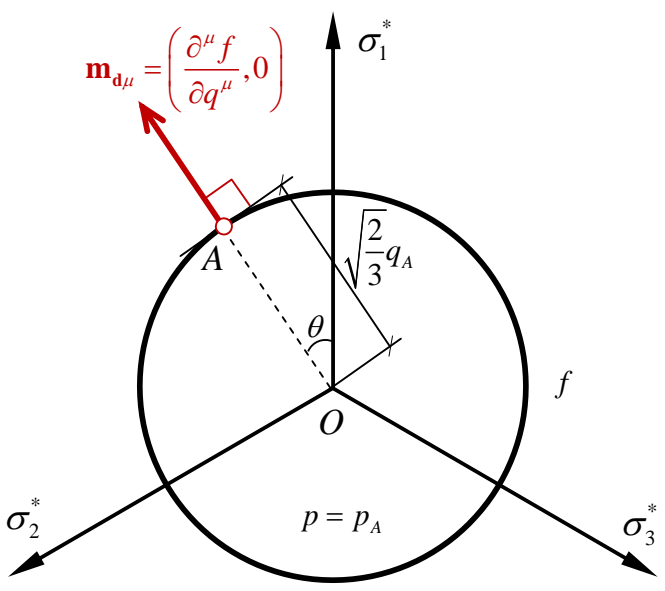

Fig. 4 Fractional plastic flow direction with the yield function constructed:

(a) by $p, q$ and $\theta$ in the meridian plane; (b) by $p, q$ and $\theta$ in the deviatoric plane;

(c) only by $q$ and $\theta$ in the meridian plane; (d) only by $p$ and $q$ in the deviatoric plane. 


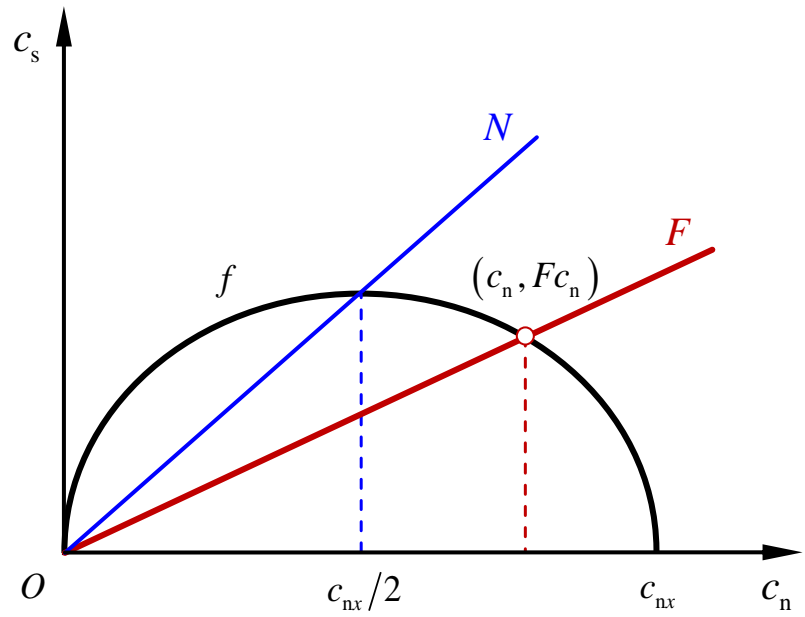

631

Fig. 5 Yield surface in the meridian plane 
632

633

634

635

636

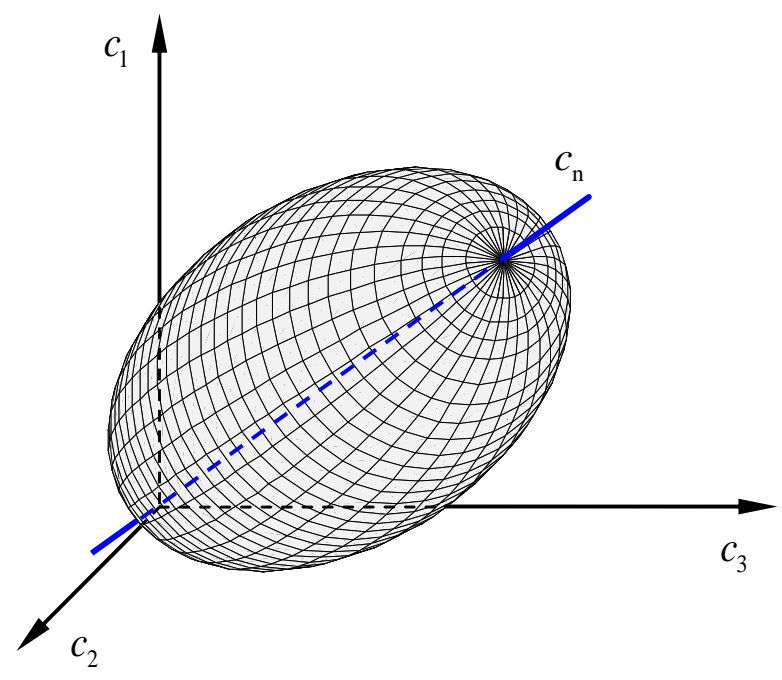

(a)

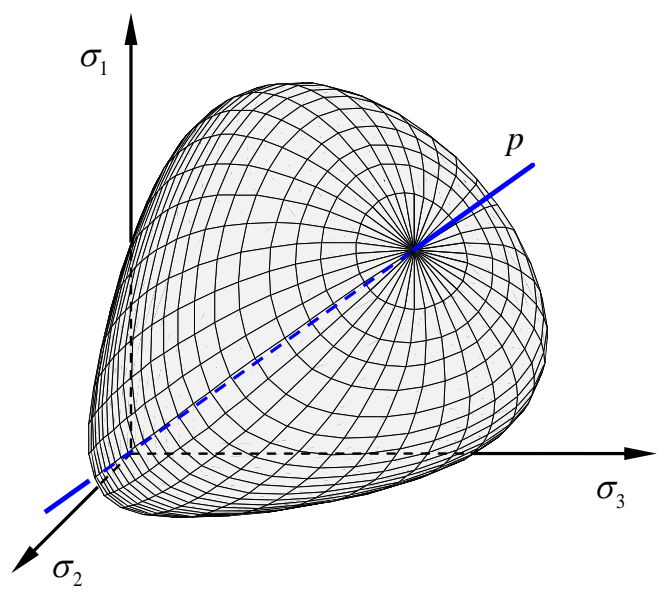

(b)

Fig. 6 Yield surface: (a) in the $c_{i j}$ space; (b) in the $\sigma_{i j}$ space. 


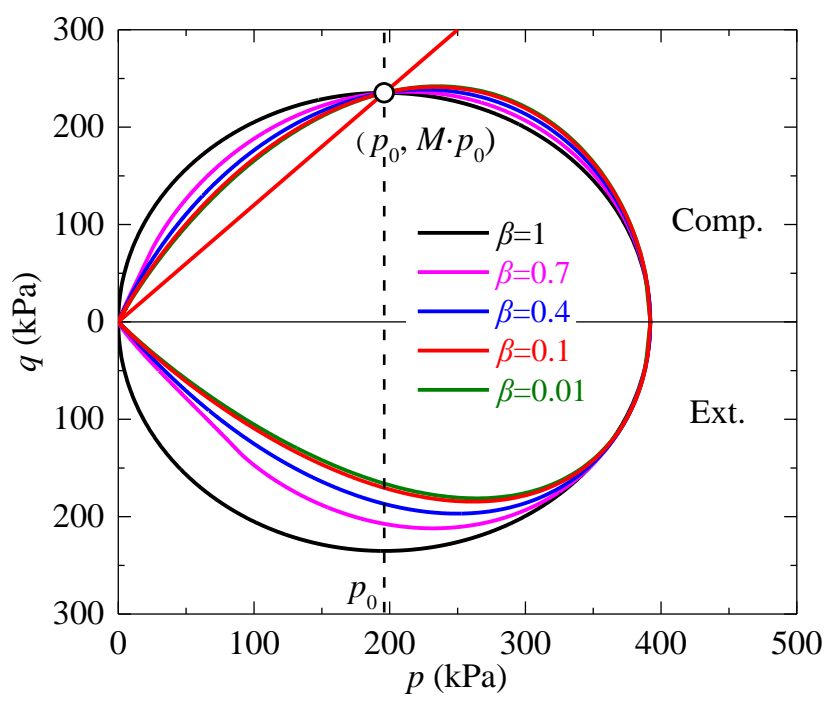

638

(a)

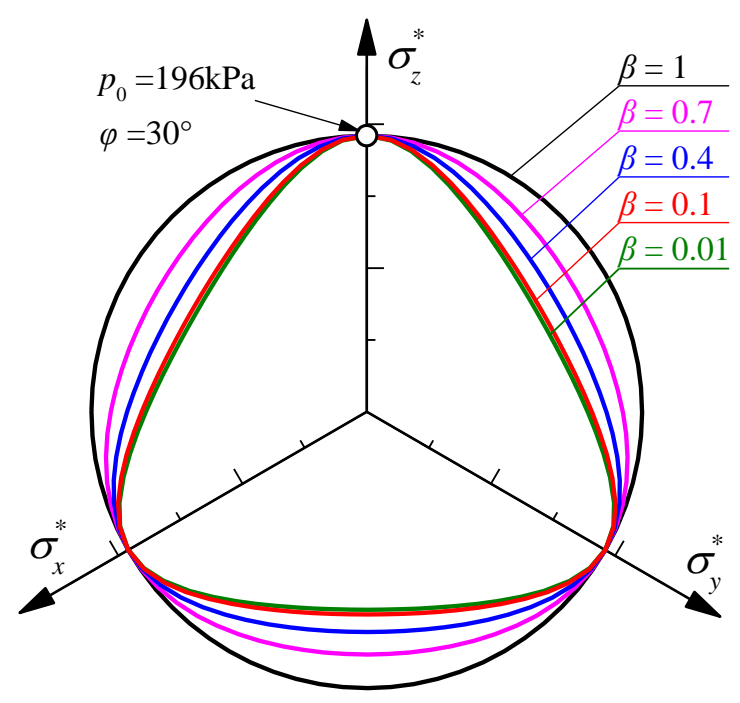

(b)

Fig. 7 Yield curves in the $\sigma_{i j}$ space with the decrease of $\beta$ :

(a) in the meridian plane; (b) in the deviatoric plane. 


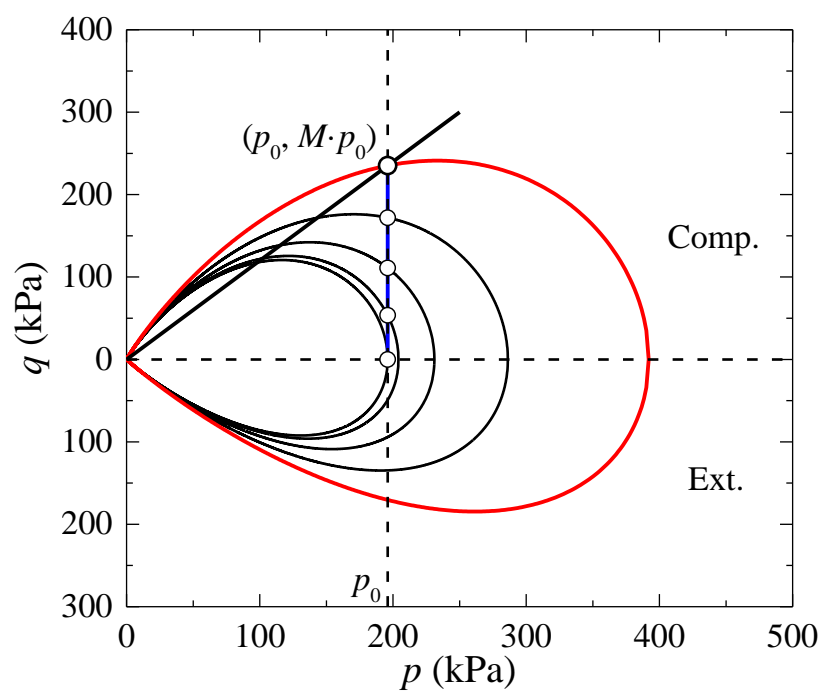

644

(a)

645

646

647

648

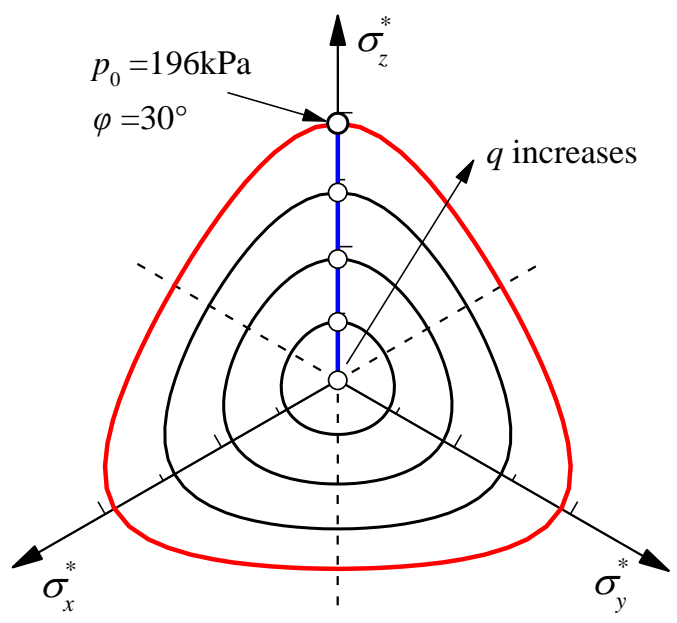

(b)

Fig. 8 Yield curve in the $\sigma_{i j}$ space with the increase of $q$ at a constant $p$ :

(a) in the meridian plane; (b) in the deviatoric plane. 
649

650

651

652

653

654

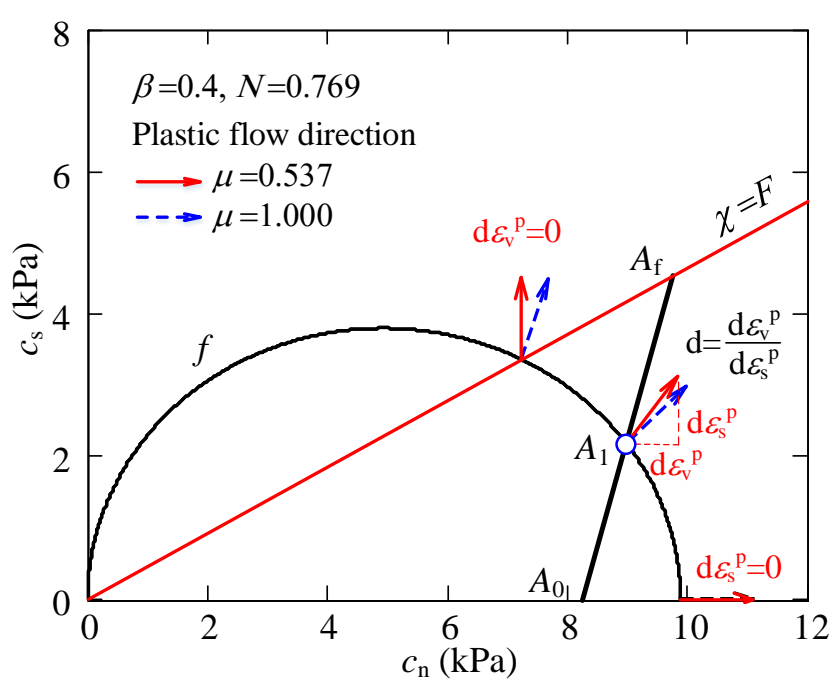

(a)

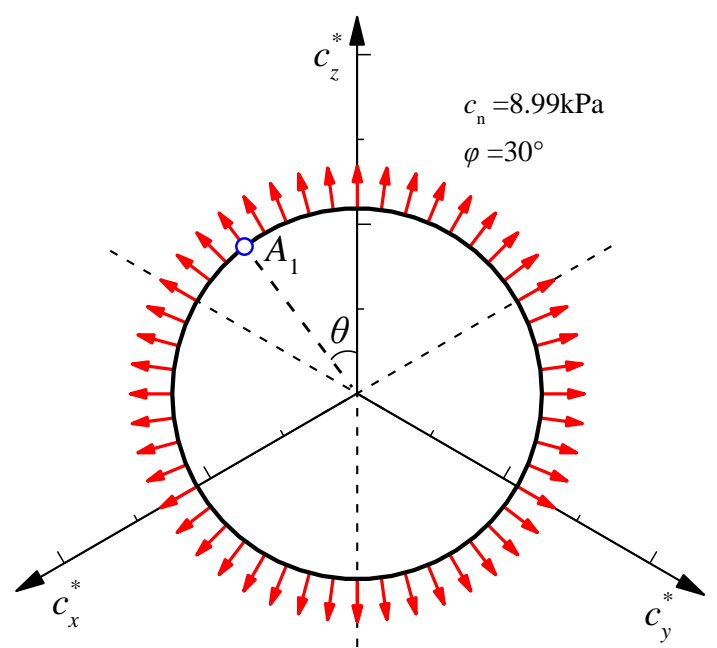

(b)

Fig. 9 Plastic flow direction in the $c_{i j}$ space at the current loading point:

(a) in the meridian plane with $\theta=\theta_{A}$; (b) in the deviatoric plane with $p=p_{A}$. 


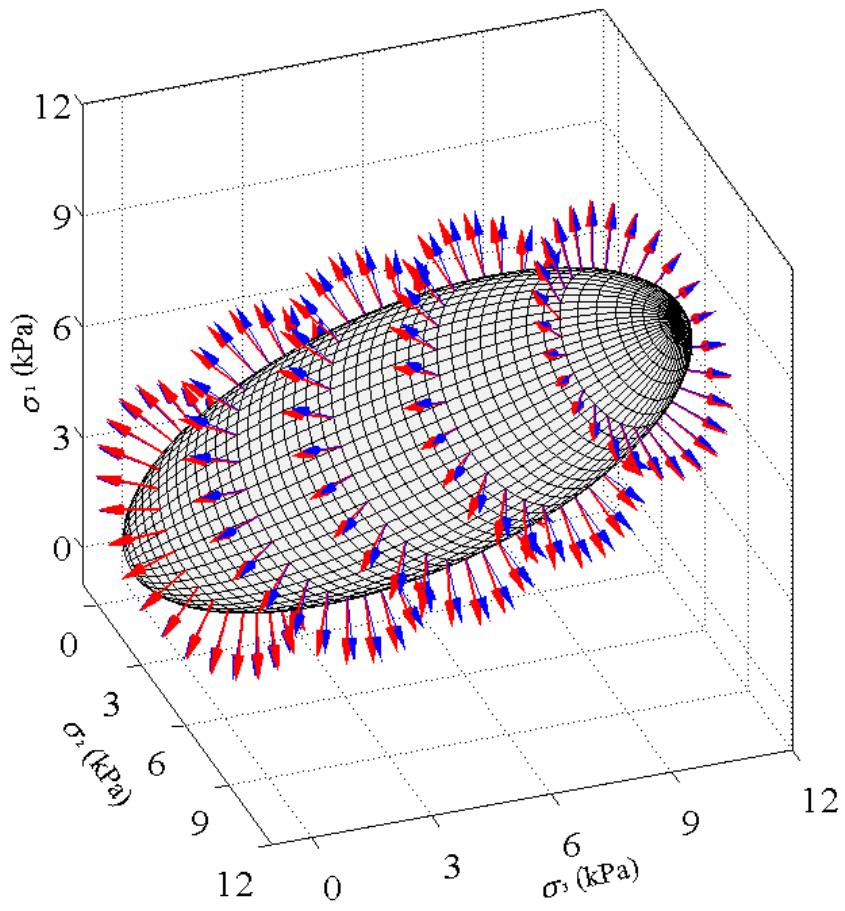

656

Fig. 10 Plastic flow direction in the $c_{i j}$ space. 


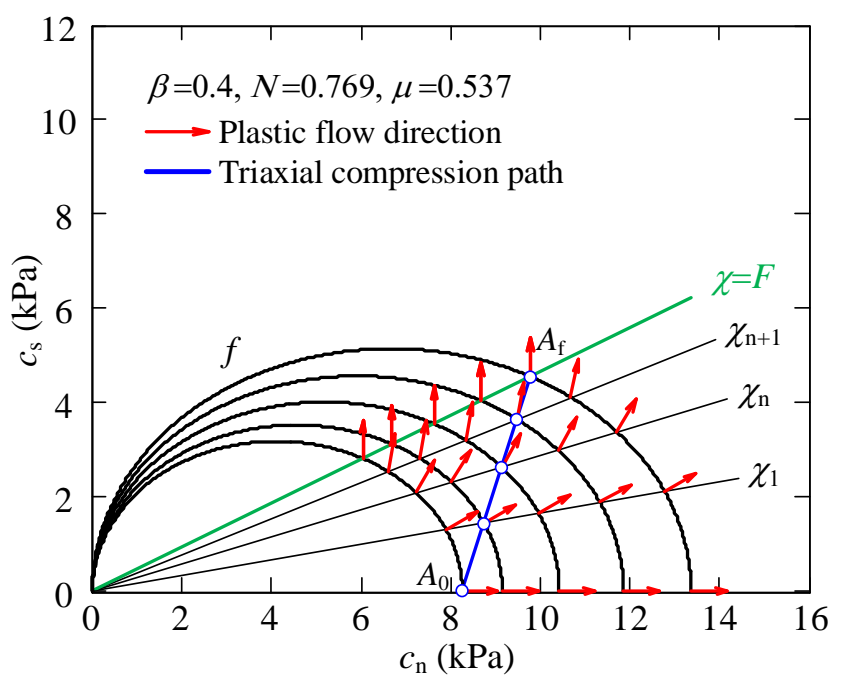

Fig. 11 Plastic flow direction during the loading process. 


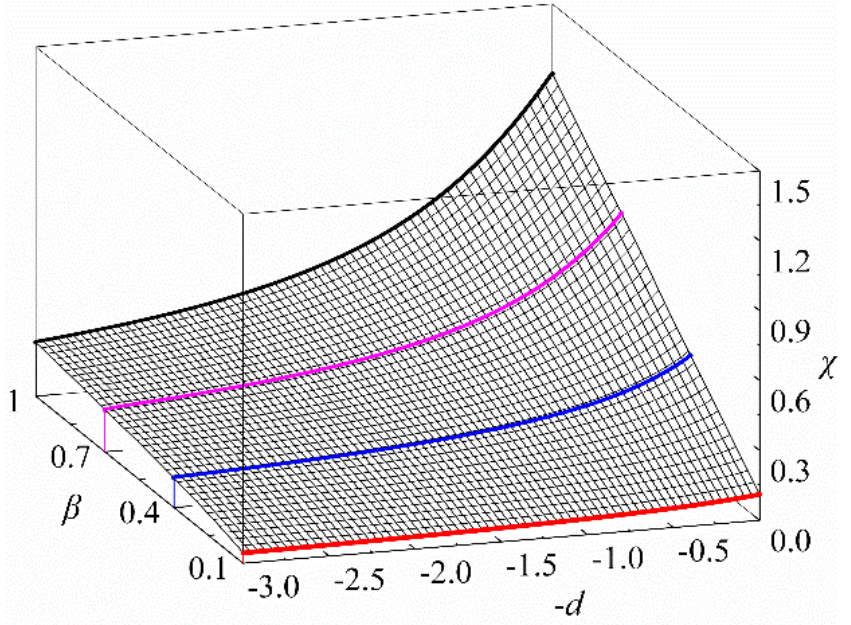

660

(a)

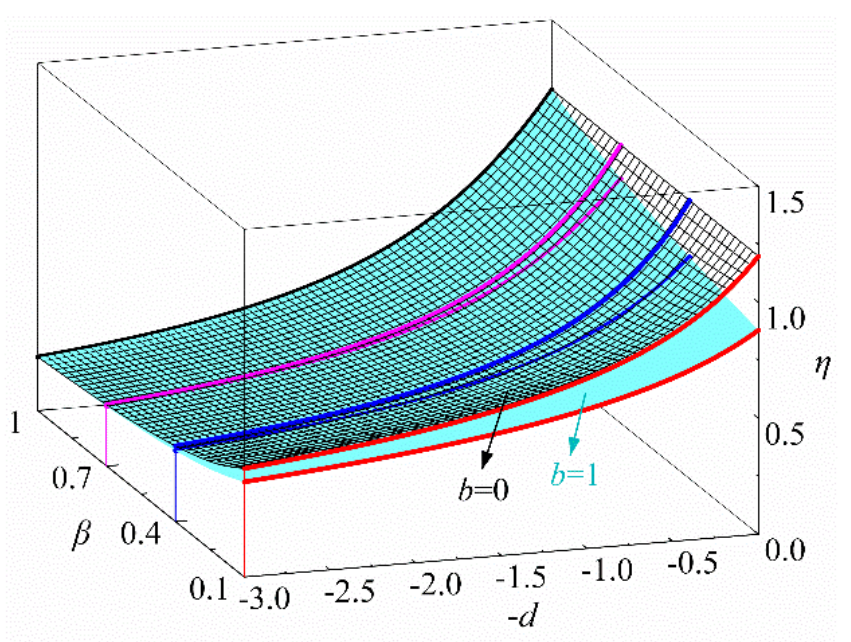

661

(b)

Fig. 12 Stress-dilatancy relationship for different $\beta$-values: (a) in the $c_{i j}$ space; (b) in the $\sigma_{i j}$ space. 


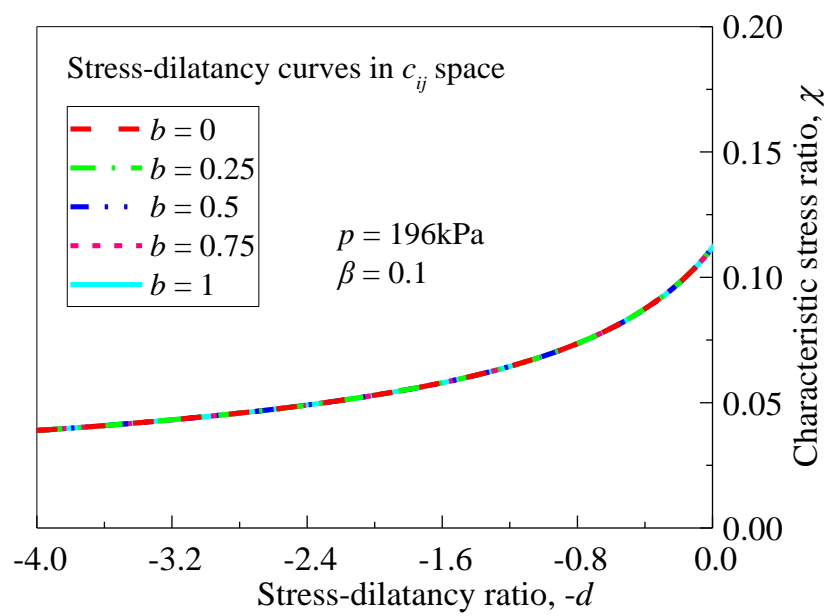

(a)

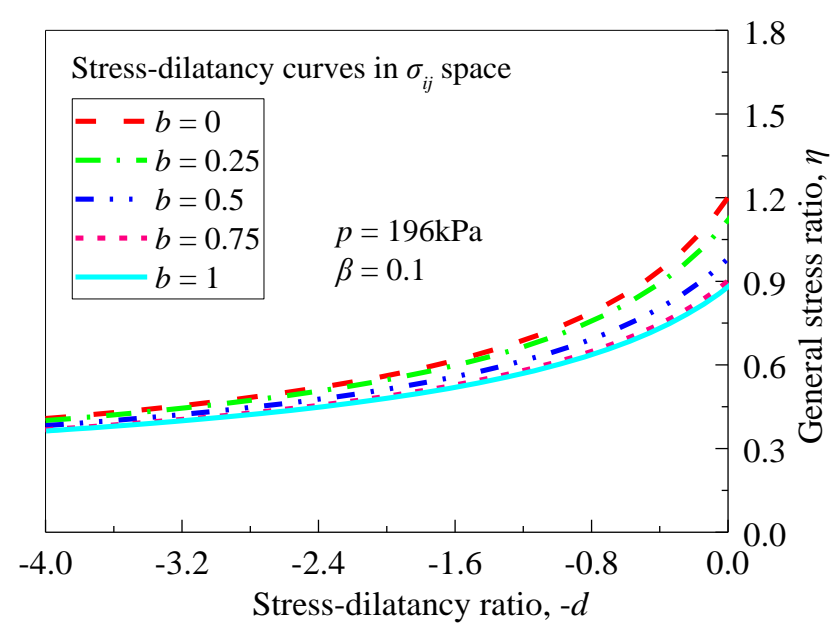

Fig. 13 Stress-dilatancy curves when $\beta=0.1$ : (a) in the $c_{i j}$ space; (b) in the $\sigma_{i j}$ space. 


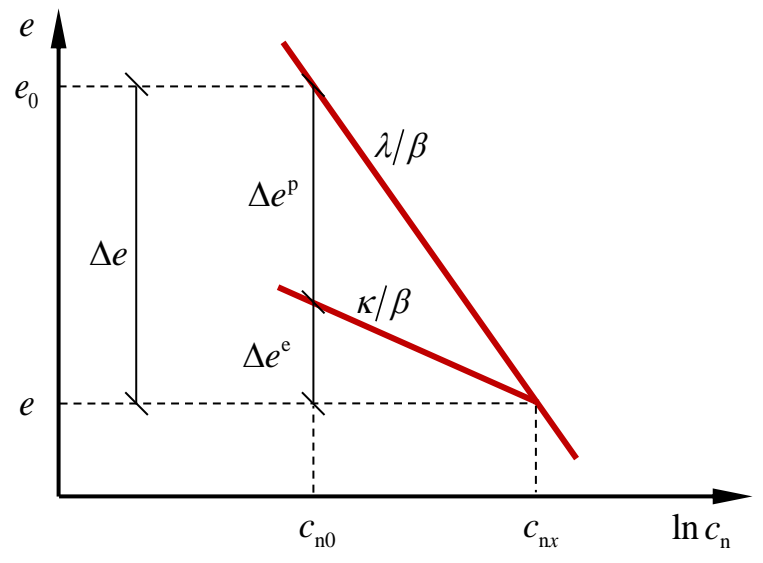

671

Fig. $14 e-\ln c_{\mathrm{n}}$ relationship in the $c_{i j}$ space. 


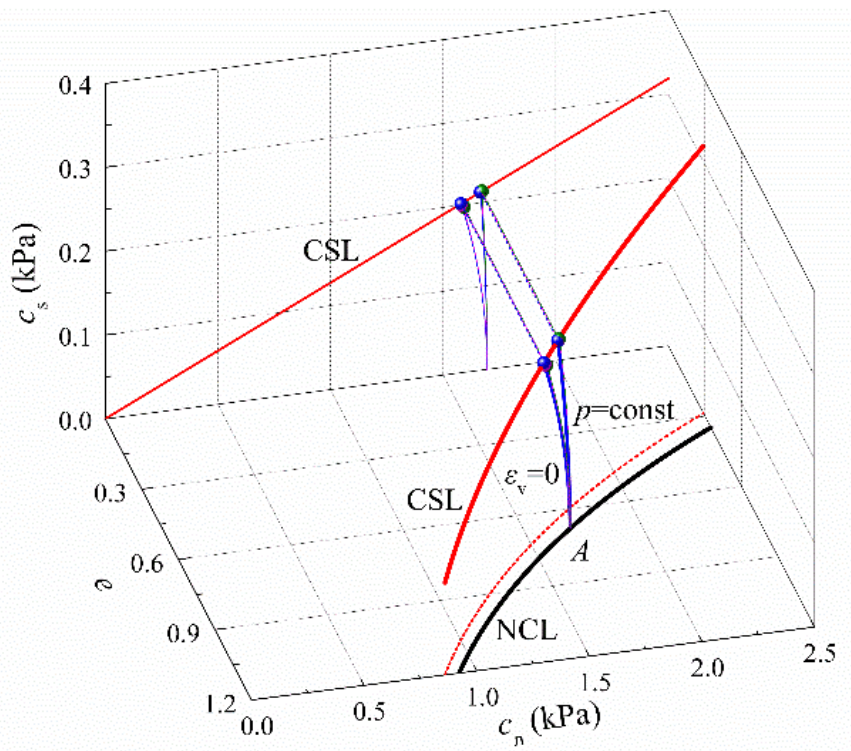

673

(a)

674

675

676

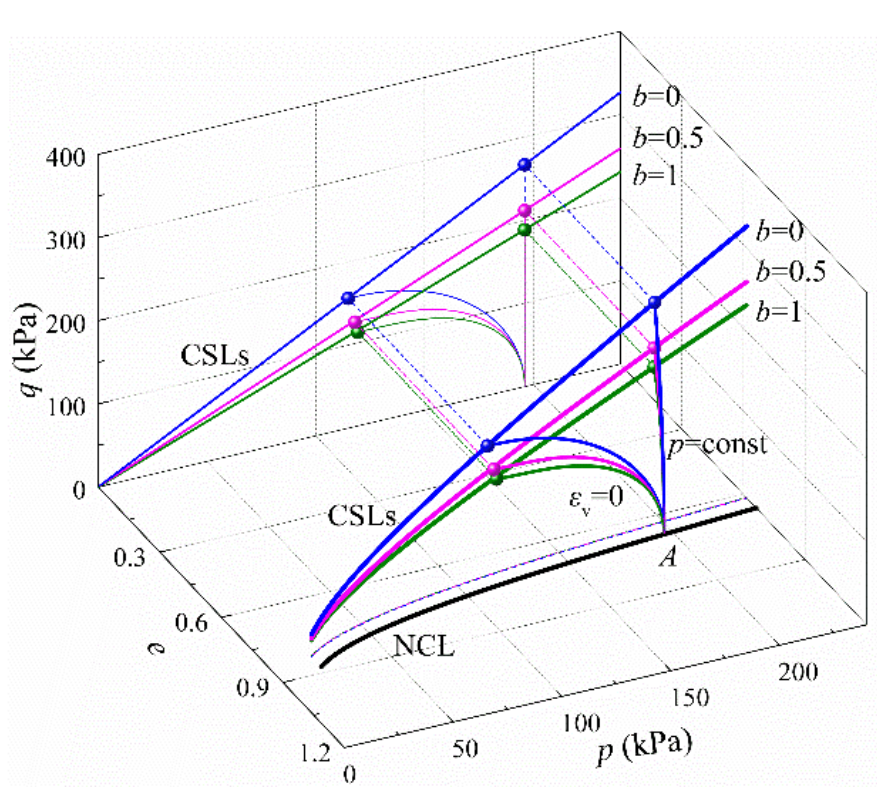

(b)

Fig. 15 Stress paths and critical state lines: (a) in the $c_{i j}$ space; (b) in the $\sigma_{i j}$ space. 


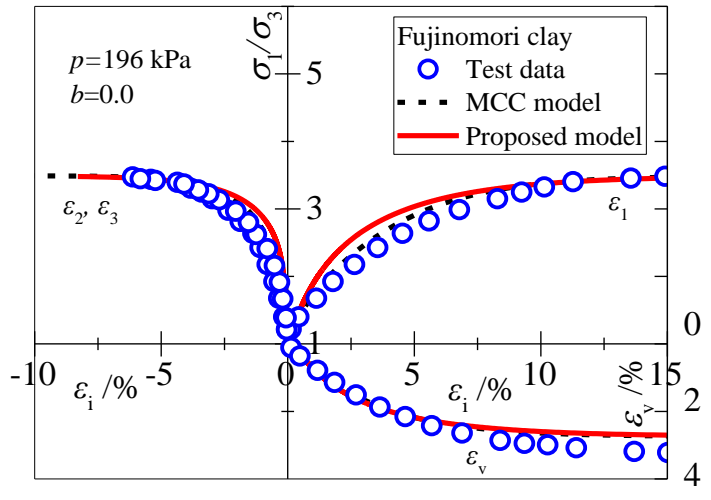

(a)

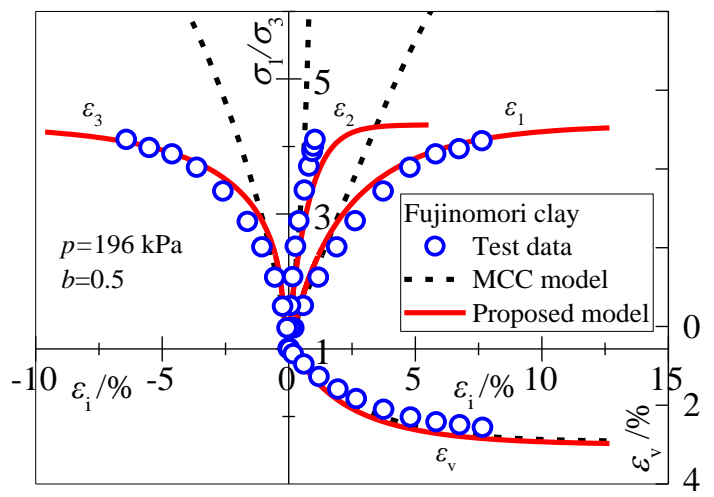

(c)

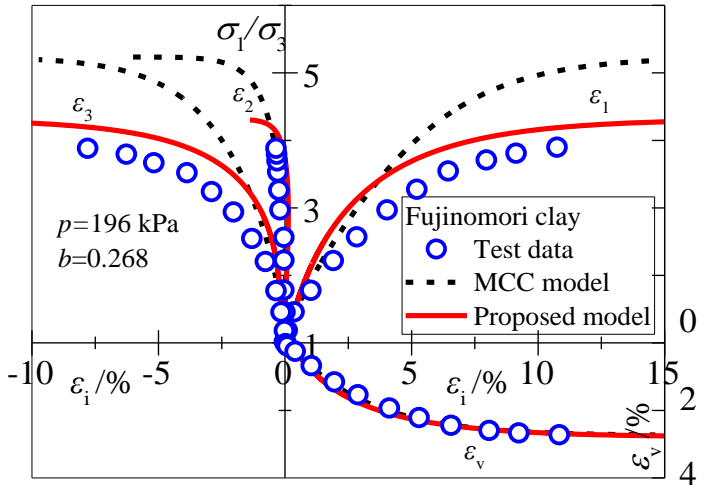

(b)
679

680

681

682

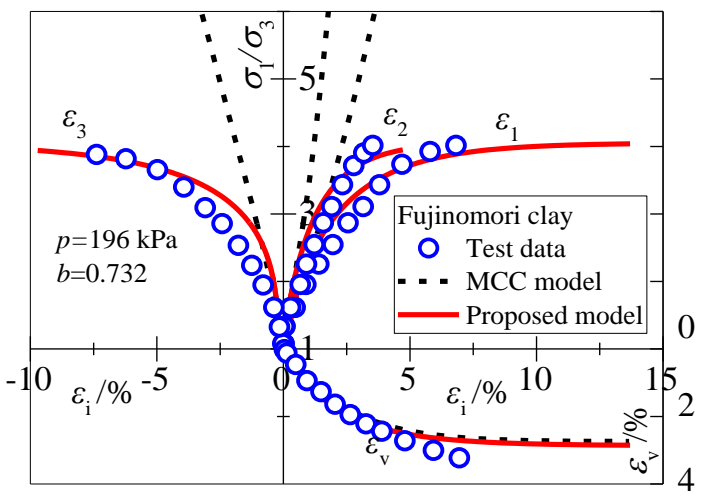

(d)

Fig. 16 Comparison between test results (data from Chowdhury \& Nakai, 1998) and predicted results under true 3D stress conditions at $p=196 \mathrm{kPa}$ with: (a) $b=0$; (b) $b=0.268$; (c) $b=0.5$; (d) $b=0.732$. 
683

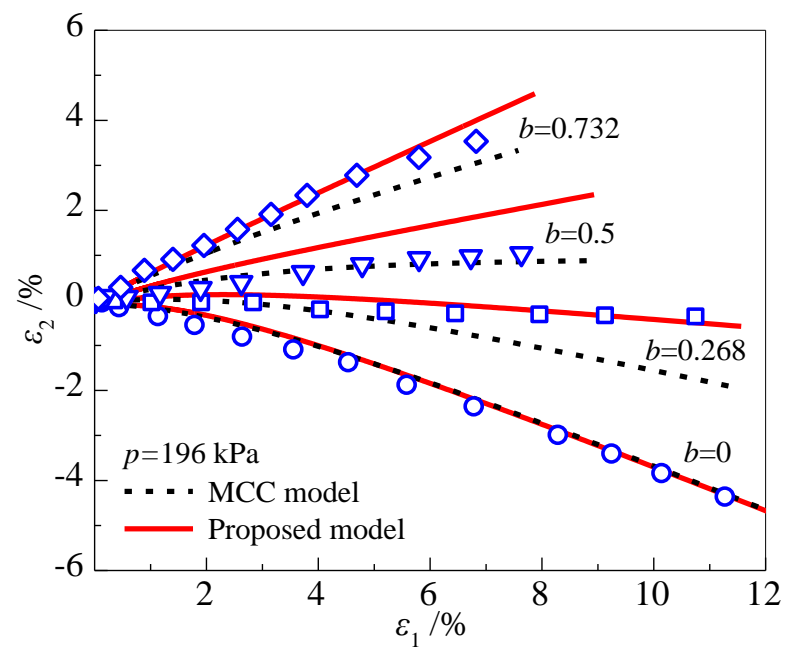

(a)

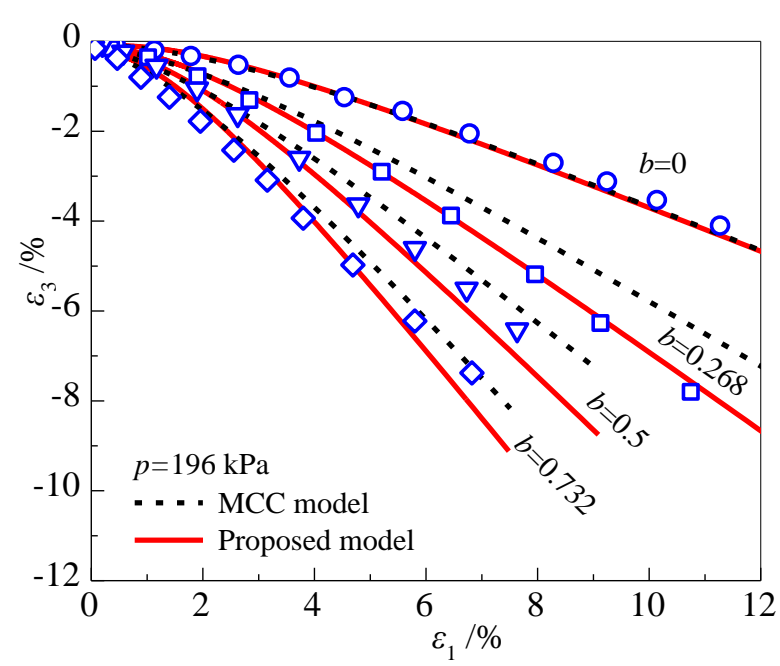

(b)

Fig. 17 Comparisons between test results (data from Chowdhury \& Nakai, 1998) and predicted results between principal stains: (a) $\varepsilon_{2}-\varepsilon_{1}$; (a) $\varepsilon_{3}-\varepsilon_{1}$. 
689

690

691

692

693

694

695

696

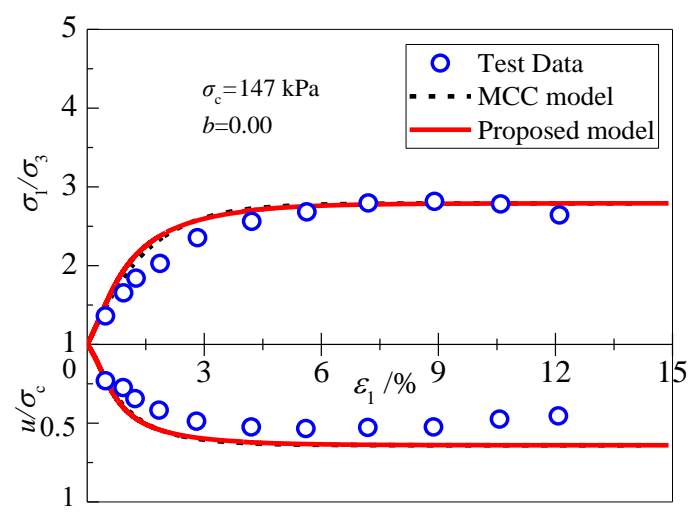

(a)

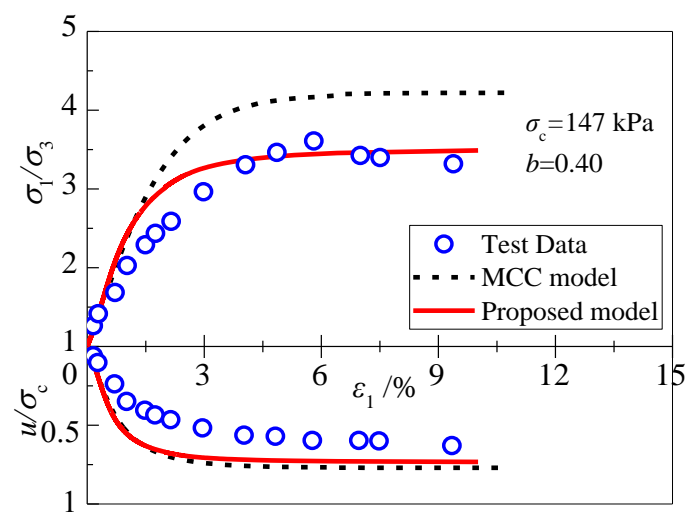

(c)

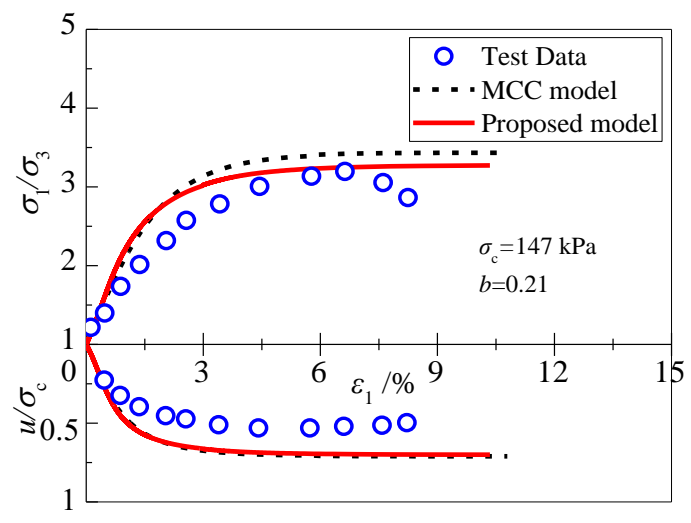

(b)

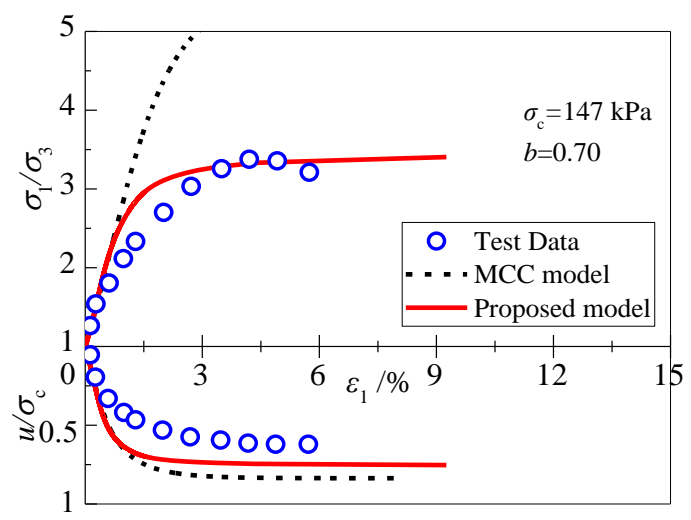

(d)

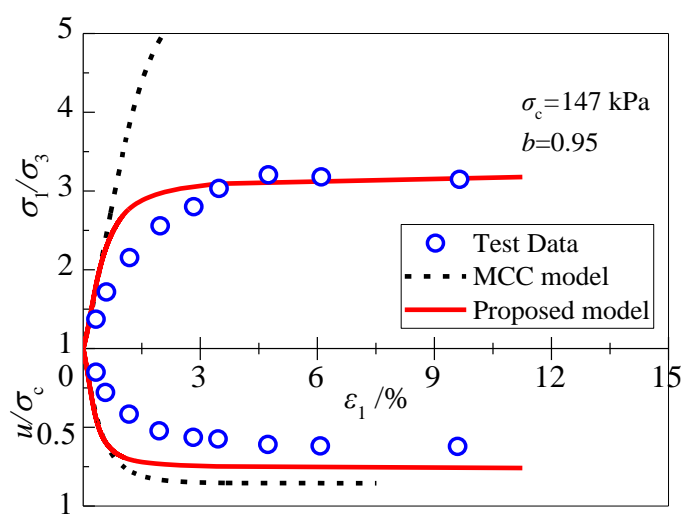

(e)

Fig. 18 Comparisons between test results (data from Lade \& Musante, 1978) and predicted results with $\sigma_{\mathrm{c}}=147 \mathrm{kPa}$ and: (a) $b=0$; (b) $b=0.21$; (b) $b=0.40$; (d) $b=0.70$; (e) $b=0.95$. 
697

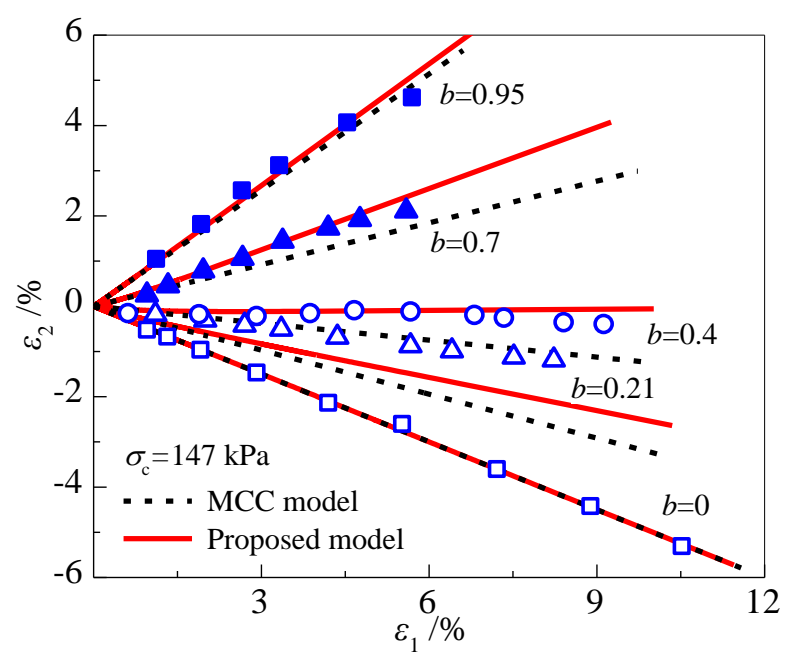

(a)

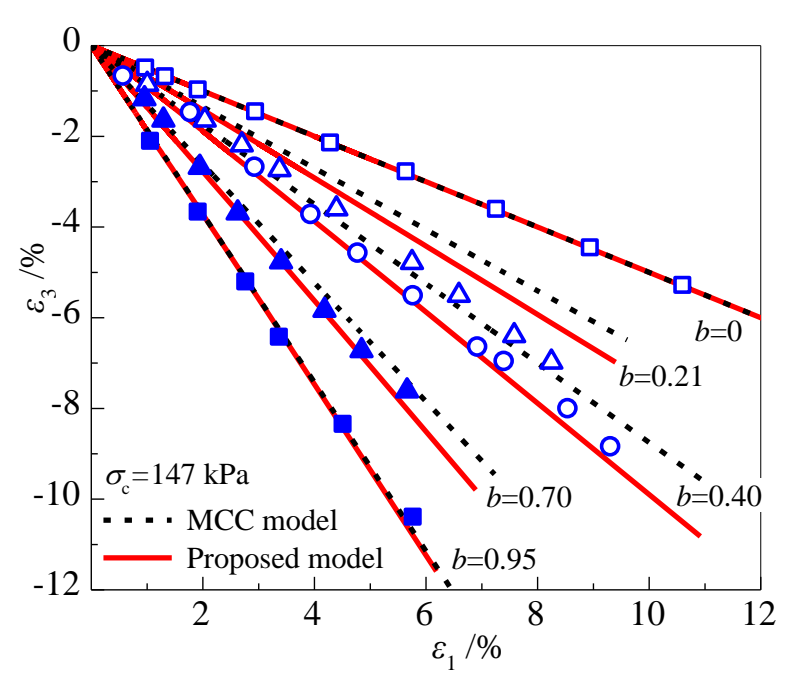

(b)

Fig. 19 Comparisons between test results (data from Lade \& Musante, 1978) and predicted results between principal stains: (a) $\varepsilon_{2}-\varepsilon_{1}$; (b) $\varepsilon_{3}-\varepsilon_{1}$.

\section{REFERENCES}

Borja, R. I., Sama, K. M., and Sanz, P. F., 2003. On the numerical integration of three-invariant elastoplastic constitutive models. Comput. Methods Appl. Mech. Eng. 192 (9-10), 1227-1258.

Chowdhury, E. Q., and Nakai, T., 1998. Consequences of the $t_{i j}$-concept and a new modeling approach. Comput. Geotech. 23 (3), 131-164.

Colinas-Armijo, N., Paola, M. D., and Pinnola, F. P., 2016. Fractional characteristic times and dissipated energy in fractional linear viscoelasticity. Commun. Nonlinear Sci. 37, 14-30.

Collins, I. F., 2003. A systematic procedure for constructing critical state models in three dimensions. 
Int. J. Solids Struct. 40 (17), 4379-4397.

Collins, I. F., and Houlsby, G. T., 1997. Application of thermomechanical principles to the modelling of geotechnical materials. Proceedings of the Royal Society a: Mathematical, Physical and Engineering Sciences. 453 (1964), 1975-2001.

Duriez, J., and Vincens, É., 2015. Constitutive modelling of cohesionless soils and interfaces with various internal states: an elasto-plastic approach. Comput. Geotech. 63, 33-45.

Gao, Z. W., and Zhao, J. D., 2017. A non-coaxial critical-state model for sand accounting for fabric anisotropy and fabric evolution. Int. J. Solids Struct. 106-107, 200-212.

Khalili, N., and Liu, M. D., 2008. On generalization of constitutive models from two dimensions to three dimensions. Int. J. Numer. Anal. Met. 32 (17), 2045-2065.

Lade, P. V., and Musante, H. M., 1978. Three-dimensional behavior of remolded clay. Journal of the Geotechnical Engineering Division, ASCE. 104 (2), 193-209.

Lade, P. V., Nelson, R. B., and Ito, Y. M., 1987. Nonassociated flow and stability of granular materials. J. Eng. Mech, ASCE. 113 (9), 1302-1318.

Li, X. S., and Dafalias, Y. F., 2000. Dilatancy for cohesionless soils. Géotechnique. 50 (4), 449-460.

Lu, D. C., Liang, J. Y., Du, X. L., Wang, G. S., and Shire, T., 2018. A novel transversely isotropic strength criterion for soils based on a mobilised plane approach. Géotechnique, https://doi.org/10.1680/jgeot.17.P.191.

Lu, D. C., Ma, C., Du, X. L., Jin, L., and Gong, Q. M., 2017. Development of a new nonlinear unified strength theory for geomaterials based on the characteristic stress concept. International Journal of Geomechanics, ASCE. 17 (2), 4016058.

Lu, D. C., Du, X. L., Wang, G. S., Zhou, A. N., and Li, A. K., 2016. A three-dimensional elastoplastic constitutive model for concrete. Computers and Structures. 163, 41-55.

Ma, C., Lu, D. C., Du, X. L., and Zhou, A. N., 2017. Developing a 3D elastoplastic constitutive model for soils: a new approach based on characteristic stress. Comput. Geotech. 86, 129-140.

Mashayekhi, S., Miles, P., Hussaini, M. Y., and Oates, W. S., 2018. Fractional viscoelasticity in fractal and non-fractal media: theory, experimental validation, and uncertainty analysis. J. Mech. Phys. Solids. $111,134-156$.

Meng, R., Yin, D., Zhou, C., and Wu, H., 2016. Fractional description of time-dependent mechanical property evolution in materials with strain softening behavior. Appl. Math. Model. 40 (1), 398-406.

Mortara, G., 2015. A constitutive framework for the elastoplastic modelling of geomaterials. Int. J. Solids Struct. 63 (6), 139-152.

Muir Wood, D. M., 1990. Soil behaviour and critical state soil mechanics. Cambridge University Press.

Nakai, T., Shahin, H. M., Kikumoto, M., Kyokawa, H., Zhang, F., and Farias, M. M., 2011. A simple and unified three-dimensional model to describe various characteristics of soils. Soils Found. 51 (6), 
Panteghini, A., and Lagioia, R., 2018. An extended modified Cam-clay yield surface for arbitrary meridional and deviatoric shapes retaining full convexity and double homothety. Géotechnique. 68 (7), 590-601.

Podlubny, I., 1998. Fractional differential equations: an introduction to fractional derivatives fractional differential equations to methods of their solution and some of their applications. Academic Press.

Roscoe, K. H., and Burland, J. B. On the generalised stress-strain behaviour of 'wet' clay. In: Heyman, J., and Leckie, F. A., eidtors. Engineering Plasticity. Cambridge at the University Press; 1968, pp. 535-609.

Sumelka, W., 2014. Fractional viscoplasticity. Mech. Res. Commun. 56, 31-36.

Sumelka, W., and Nowak, M., 2016. Non-normality and induced plastic anisotropy under fractional plastic flow rule: a numerical study. Int. J. Numer. Anal. Met. 40 (5), 651-675.

Sun, D. A., Matsuoka, H., Yao, Y. P., and Ishii, H., 2004. An anisotropic hardening elastoplastic model for clays and sands and its application to FE analysis. Comput. Geotech. 31 (1), 37-46.

Sun, Y. F., Indraratna, B., Carter, J. P., Marchant, T., and Nimbalkar, S., 2017. Application of fractional calculus in modelling ballast deformation under cyclic loading. Comput. Geotech. 82, 16-30.

Sun, Y. F., and Xiao, Y., 2017. Fractional order plasticity model for granular soils subjected to monotonic triaxial compression. Int. J. Solids Struct. 118-119, 224-234.

Sun, Y. F., Gao, Y. F., and Shen, Y., 2018a. Mathematical aspect of the state-dependent stress-dilatancy of granular soil under triaxial loading. Géotechnique., https://doi.org/10.1680/jgeot.17.T.029.

Sun, Y. F., Gao, Y. F., and Zhu, Q. Z., 2018b. Fractional order plasticity modelling of state-dependent behaviour of granular soils without using plastic potential. Int. J. Plasticity. 102 (3), 53-69.

Wang, G. S., Lu, D. C., Du, X. L., Zhou, X., and Cao, S. T., 2018. A true 3d frictional hardening elastoplastic constitutive model of concrete based on a unified hardening/softening function. J. Mech. Phys. Solids. 119, 250-273.

Xiao, Y., and Liu, H. L., 2017. Elastoplastic constitutive model for rockfill materials considering particle breakage. Int. J. Geomech. 17 (1), 4016041.

Xiao, Y., Liu, H. L., Chen, Y. M., and Jiang, J. S., 2004. Bounding surface plasticity model incorporating the state pressure index for rockfill materials. J. Eng. Mech, ASCE. 141 (11), 4014087.

Yao, Y. P., Hou, W., and Zhou, A. N., 2009. UH model: three-dimensional unified hardening model for overconsolidated clays. Géotechnique. 59 (5), 451-469.

Yao, Y. P., Lu, D. C., Zhou, A. N., and Zou, B., 2004. Generalized non-linear strength theory and transformed stress space. Science in China Ser. E. 47 (6), 691-709.

Yu, H. S., 1998. CASM: a unified state parameter model for clay and sand. Int. J. Numer. Anal. Met. 22 (8), 621-653. 

anisotropy. Géotechnique. 63 (8), 695-704.

784 Zhou, A. N., and Sheng, D. C., 2015. An advanced hydro-mechanical constitutive model for unsaturated soils with different initial densities. Comput. Geotech. 63, 46-66.

786

Zhou, W., Liu, J. Y., Ma, G., and Chang, X. L., 2017. Three-dimensional DEM investigation of critical

787 state and dilatancy behaviors of granular materials. Acta Geotech. 12 (3), 527-540. 